ARTICLE

DOI: $10.1038 / \mathrm{s} 41467-017-00942-5$

\title{
Survival of pancreatic cancer cells lacking KRAS function
}

Mandar Deepak Muzumdar ${ }^{1,2,3}$, Pan-Yu Chen ${ }^{1,4}$, Kimberly Judith Dorans ${ }^{1}$, Katherine Minjee Chung ${ }^{1}$, Arjun Bhutkar ${ }^{1}$, Erin Hong ${ }^{1}$, Elisa M. Noll (10 5,6, Martin R. Sprick (iD ${ }^{5,6}$, Andreas Trumpp ${ }^{5,6}$ \& Tyler Jacks ${ }^{1,4,7}$

Activating mutations in the proto-oncogene KRAS are a hallmark of pancreatic ductal adenocarcinoma (PDAC), an aggressive malignancy with few effective therapeutic options. Despite efforts to develop KRAS-targeted drugs, the absolute dependence of PDAC cells on KRAS remains incompletely understood. Here we model complete KRAS inhibition using CRISPR/Cas-mediated genome editing and demonstrate that KRAS is dispensable in a subset of human and mouse PDAC cells. Remarkably, nearly all KRAS deficient cells exhibit phosphoinositide 3-kinase (PI3K)-dependent mitogen-activated protein kinase (MAPK) signaling and induced sensitivity to PI3K inhibitors. Furthermore, comparison of gene expression profiles of PDAC cells retaining or lacking KRAS reveal a role of KRAS in the suppression of metastasis-related genes. Collectively, these data underscore the potential for PDAC resistance to even the very best KRAS inhibitors and provide insights into mechanisms of response and resistance to KRAS inhibition.

\footnotetext{
${ }^{1}$ David H. Koch Institute for Integrative Cancer Research, Massachusetts Institute of Technology, Cambridge, MA 02139, USA. ${ }^{2}$ Dana-Farber Cancer Institute, Boston, MA 02215, USA. ${ }^{3}$ Harvard Medical School, Boston, MA 02215, USA. ${ }^{4}$ Department of Biology, Massachusetts Institute of Technology, Cambridge, MA 02139, USA. ${ }^{5}$ Heidelberg Institute for Stem Cell Technology and Experimental Medicine (HI-STEM), Heidelberg 69120, Germany. ${ }^{6}$ Division of Stem Cells and Cancer, German Cancer Research Center (DKFZ), Heidelberg 69120, Germany. ${ }^{7}$ Howard Hughes Medical Institute, Massachusetts Institute of Technology, Cambridge, MA 02139, USA. Mandar Deepak Muzumdar and Pan-Yu Chen contributed equally to this work. Correspondence and requests for materials should be addressed to T.J. (email: tjacks@mit.edu)
} 
$\mathrm{P}$ ancreatic ductal adenocarcinoma (PDAC) is the third leading cause of cancer death in the United States and a major cause of morbidity and mortality worldwide ${ }^{1,} 2$. While advances in combination chemotherapy have improved median survival ${ }^{3,}$, , long-term survival remains poor $^{1,2}$, highlighting the need for novel therapeutic approaches.
Genomic studies have identified mutations in the protooncogene KRAS as a hallmark of PDAC, occurring in $>90 \%$ of cases $^{5-8}$. KRAS is a small GTPase that acts as a molecular switch to regulate proliferation, differentiation, metabolism, and survival ${ }^{9}$. Oncogenic forms of KRAS harboring mutations in codons 12, 13, and 61 are insensitive to GTPase activating protein a

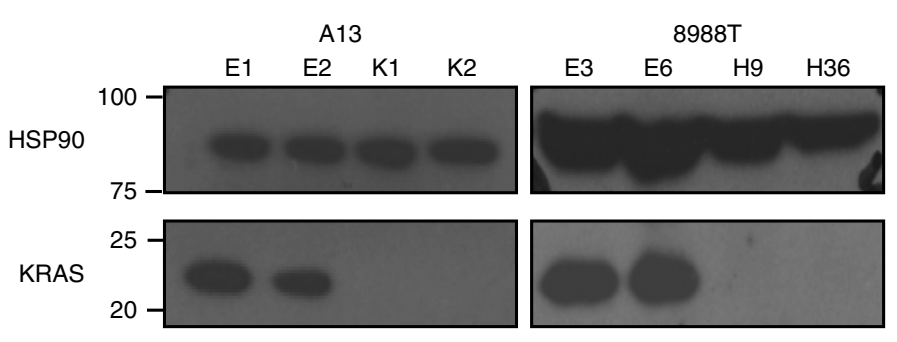

b

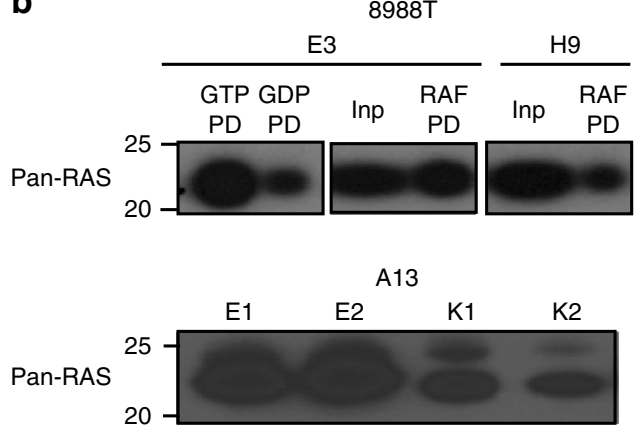

C

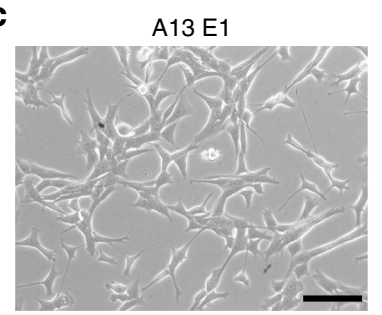

A13 K2

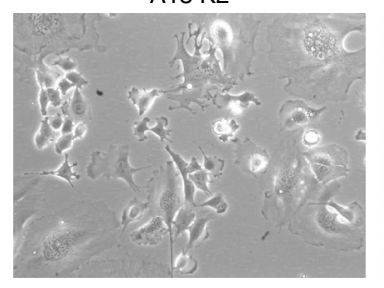

e
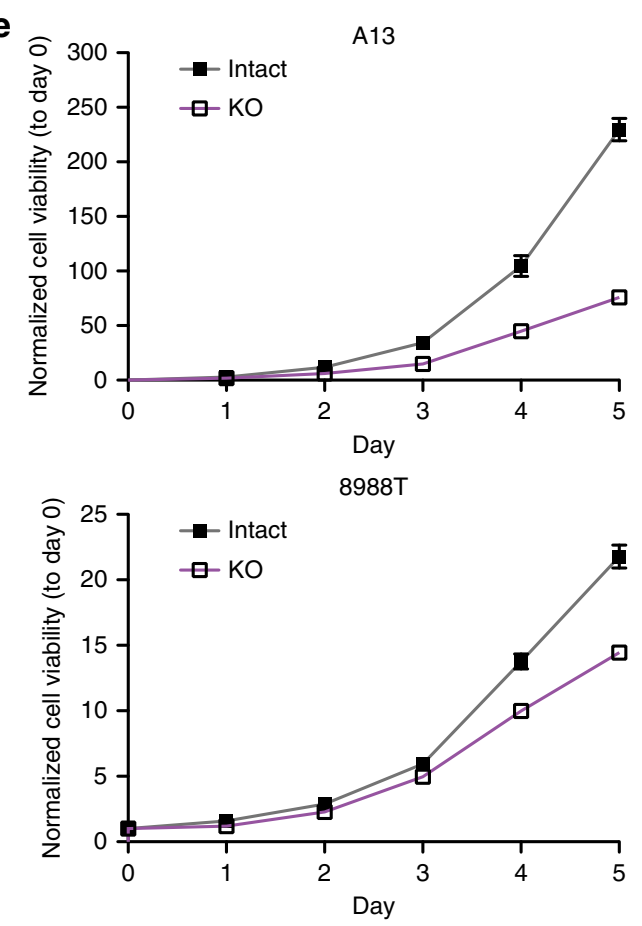

8988T E3

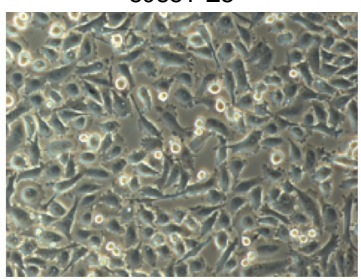

8988T H36

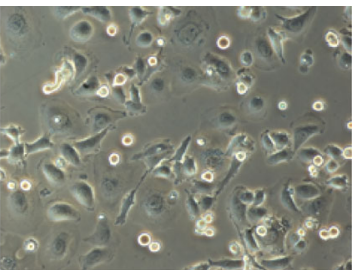

d

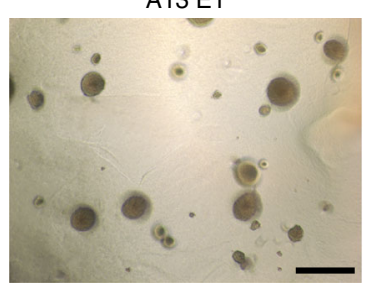

A13 K2
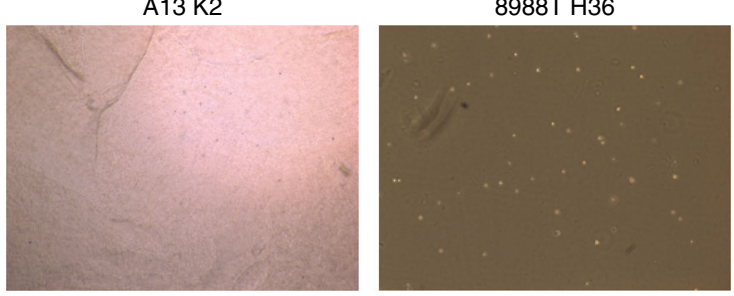

$\mathbf{f}$

\begin{tabular}{ccc}
\multicolumn{3}{c}{$\begin{array}{c}\text { Tumors developed/ } \\
\text { tumors transplanted (\%) }\end{array}$} \\
\hline Cell line & Intact & KO \\
\hline A13 & $63 / 64(98)$ & $62 / 64(97)$ \\
8988T & $8 / 16(50)$ & $10 / 16(63)$ \\
PANC-1 & $4 / 8(50)$ & $2 / 4(50)$ \\
\hline
\end{tabular}

$\mathbf{g}$

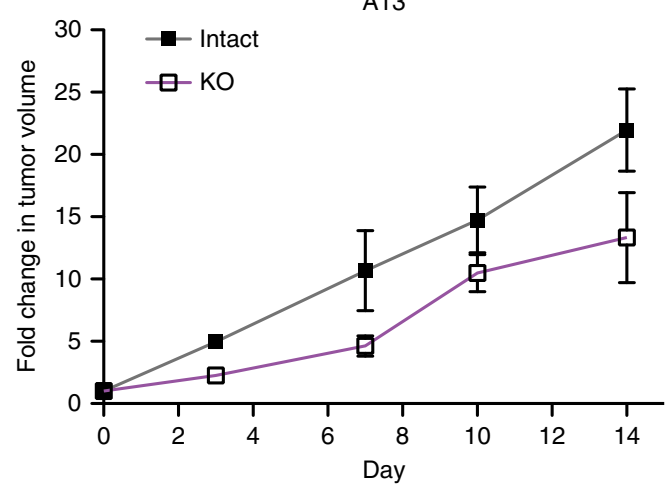


(GAP)-induced GTP hydrolysis, leading to constitutive activation ${ }^{10}$. Studies in animal models have confirmed an important role of oncogenic KRAS in tumor initiation ${ }^{11}$, making KRAS an attractive therapeutic target.

Unfortunately, the development of effective KRAS inhibitors has been hindered by several features of oncogenic KRAS: (1) its high affinity for GTP, impeding the identification of GTP-competitive inhibitors; (2) the difficulty of inducing gain-offunction hydrolytic activity with small molecules; and (3) redundant pathways for membrane localization required for KRAS activity 9,10 . New approaches to directly inhibit KRAS through covalent binding of specific mutant variants (e.g., G12C) ${ }^{12}, 13$, interference with guanine-exchange factor (GEF) association to prevent initial GTP loading ${ }^{14,15}$, and destabilization of additional membrane localization complexes ${ }^{16}$ continue to be developed. Furthermore, the success of a recent effort spearheaded by the National Cancer Institute of the United States to develop novel RAS-targeted therapies ${ }^{17}, 18$ requires a better understanding of the dependency of PDAC cells on KRAS as well as predicting resistance mechanisms that could develop in response to KRAS inhibition.

Given the lack of KRAS inhibitors, genetic tools have been used to evaluate the requirement of KRAS in PDAC maintenance. Acute KRAS knockdown by RNA interference (RNAi) decreased cell proliferation and/or induced apoptosis in a series of human PDAC (hPDAC) cancer cell lines ${ }^{19-21}$. Variability in apoptotic response to KRAS knockdown led to the classification of some cells as "KRAS-dependent" and others as "KRAS-independent" 20 , 21. Based on these studies, it was unclear whether the "KRAS-independent" phenotype was a consequence of the incomplete inhibitory effects of RNAi such that residual KRAS protein was sufficient to sustain cell survival and proliferation. Recent evidence for PDAC cell survival in the absence of oncogenic KRAS expression derived from a doxycycline (DOX)inducible oncogenic KRAS transgenic mouse model ${ }^{22}$. In this model, DOX treatment led to oncogenic KRAS expression in the pancreas to initiate tumorigenesis, while DOX withdrawal halted transgene expression and induced tumor regression. Interestingly, a subset of PDAC tumors recurred lacking KRAS transgene expression $^{22}$. Despite these findings, the absolute dependence of PDAC cells on endogenous KRAS, a prerequisite for the successful clinical development of novel KRAS inhibitors, remains unknown.

In this study, we examine the consequence of KRAS knockout in PDAC cells using the clustered regularly interspaced short palindromic repeats (CRISPR)/Cas system. The bacterial CRISPR/Cas adaptive immune system, modified for genome editing in mammalian cells, utilizes a single guide RNA (sgRNA) to direct the Cas9 nuclease to cleave matching double-stranded DNA (dsDNA) sequences, resulting in insertions and deletions via error-prone non-homologous end joining repair mechanisms ${ }^{23}$. We confirm the variable dependence of hPDAC cell lines based on prior RNAi studies ${ }^{20,21}$, and further isolate a subset of
hPDAC and murine PDAC (mPDAC) cells that can survive and proliferate despite the absence of endogenous KRAS function. An unbiased chemical screen identifies sensitivity to phosphoinositide 3-kinase (PI3K) inhibition in KRAS deficient cells, offering a pharmacologically tractable method to subvert resistance to KRAS blockade. Furthermore, we gain mechanistic insight into how PI3K inhibition simultaneously blocks the mitogen-activated protein kinase (MAPK) and AKT pathways to impair cap-dependent translation and cell viability in the context of KRAS ablation. Finally, gene expression profiling defines KRASregulated pathways in PDAC cells and reveals KRAS-relevant gene signatures that strongly predict survival in PDAC patients.

\section{Results}

CRISPR/Cas-mediated KRAS knockout in PDAC cells. To evaluate the dependence of PDAC cells on endogenous KRAS, we employed CRISPR/Cas technology ${ }^{23}$ to completely eliminate KRAS function. We expressed Streptococcus pyogenes Cas9 and a panel of sgRNAs targeting various KRAS exons (Supplementary Fig. 1a and Supplementary Table 1) in KRAS mutant hPDAC and mPDAC cell lines to identify sgRNAs that effectively induced KRAS protein loss (Supplementary Fig. 1b). Given the lack of unique protospacer adhesion motifs (PAM) encompassing mutant codon 12, our sgRNAs did not discriminate between wild-type and mutant forms of KRAS, modeling non-selective KRAS inhibition, which comprises the vast majority of approaches currently being evaluated to target $\mathrm{KRAS}^{18}$. The consequence of short-term CRISPR/Cas-mediated KRAS knockout mimicked the effect of short hairpin RNA (shRNA)-mediated KRAS knockdown on cell viability (Supplementary Fig. 1c, d). Consistent with published results ${ }^{20}$, previously defined "KRASindependent" cells (8988T, PANC-1) were largely insensitive to sgRNA transduction, while "KRAS-dependent" cells (8902) exhibited significantly decreased viability (Supplementary Fig. 1e).

We further generated single cell subclones to evaluate whether PDAC cells could survive in the complete absence of KRAS expression. We isolated KRAS deficient subclones from $8988 \mathrm{~T}$ hPDAC and A13 mPDAC cells (Fig. 1a and Supplementary Fig. 2c) and confirmed decreased total RAS activity (RAS-GTP) (Fig. 1b). Sanger sequencing revealed indels in the KRAS locus leading to premature stop codons or in-frame indels of important functional domains ${ }^{24}$ (Supplementary Fig. 2a, b), likely perturbing protein folding and stability. PDAC cells that survived KRAS loss exhibited perturbations of several growth characteristics. KRAS deficient cells displayed altered morphology (Fig. 1c and Supplementary Fig. 2d) comparable to that observed with KRAS knockdown (Supplementary Fig. 1d), significantly diminished anchorageindependent colony formation (Fig. 1d and Supplementary Fig. 2e), and slower proliferation in $2 \mathrm{D}$ and $3 \mathrm{D}$ culture (Fig. 1e and Supplementary Fig. 2f). Interestingly, KRAS deficient

Fig. 1 KRAS is dispensable for in vitro and in vivo proliferation of PDAC cells. a Western blot confirmed loss of KRAS protein in knockout clones (A13-K1,K2, 8988T-H9,H36) compared to intact clones (A13-E1,E2, 8988T E3, E6). HSP90 is loading control. b RAS-GTP levels were decreased in knockout (8988T$\mathrm{H} 9$ and A13-K1,K2) compared to intact (8988T E3 and A13-E1,E2) clones. GTP $\gamma$ S (non-hydrolysable)-treated positive control (GTP PD) and GDP-treated negative control (GDP PD) for 8988T E3 are shown. PD pull-down. Inp input before pull-down. c KRAS deficient clones exhibited altered cell morphology, characterized by increased cell size, cytoplasmic translucency, and smooth edges. Scale bar is $100 \mu \mathrm{m}$. d KRAS deficient clones showed diminished anchorage-independent growth in soft agar. Scale bar is $500 \mu \mathrm{m}$. e Growth curves for A13 and $8988 \mathrm{~T}$ KRAS intact and deficient (KO) clones. Average cell viability (normalized to day 0 ) \pm s.e.m. is plotted for A13 ( $n=2$ clones) and 8988 T ( $n=4$ clones). f A13, 8988T, and PANC- 1 clones exhibited comparable efficiency generating tumors following subcutaneous transplant in nude mice regardless of KRAS status. Shown are cumulative data from two KRAS intact and two deficient clones for A13 and 8988T and one intact and one deficient clone for PANC-1. g A13 KRAS deficient tumors grew at a slower rate than intact tumors. Average tumor volume fold increase (normalized to day 0 when tumors were $\sim 0.5 \mathrm{~cm}$ in diameter) $\pm \mathrm{s}$.e.m. is plotted ( $n=8$ tumors per group) 
cells retained the ability to form subcutaneous tumors in immunocompromised mice (Fig. 1f), though tumors grew more slowly (Fig. 1g).
To ensure that the observed phenotypes were due to on-target KRAS mutagenesis, we sequenced the closest exonic sgRNA mismatches and found no off-target mutations in these loci

a
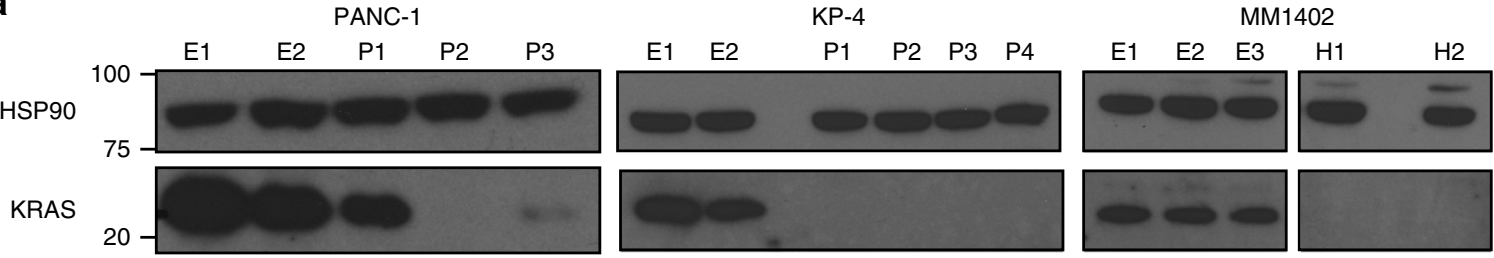

b
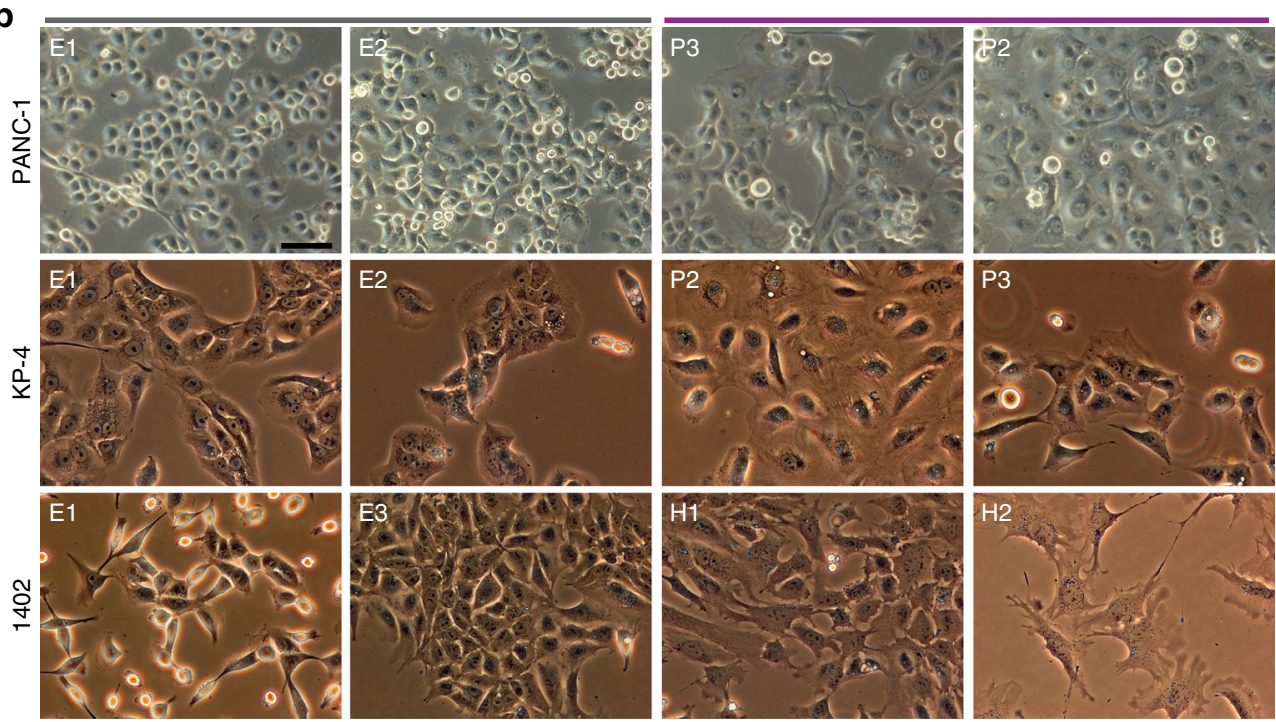

C

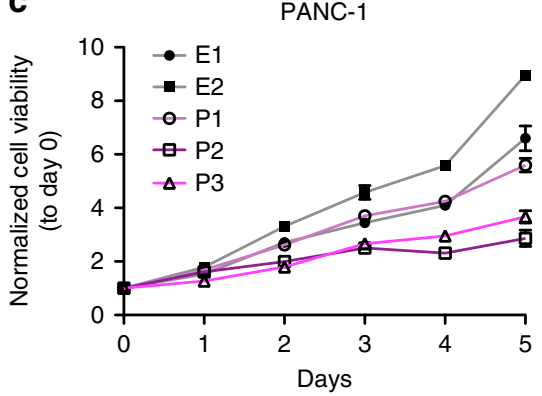

d
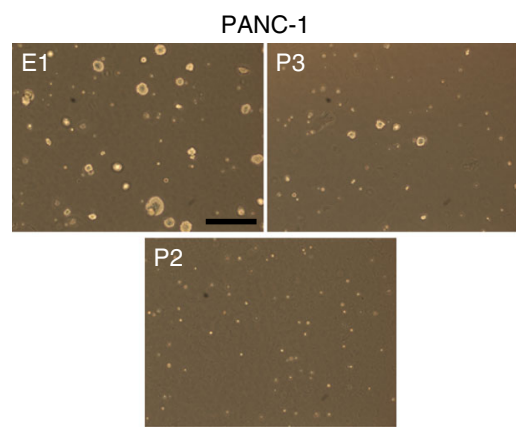

KP4

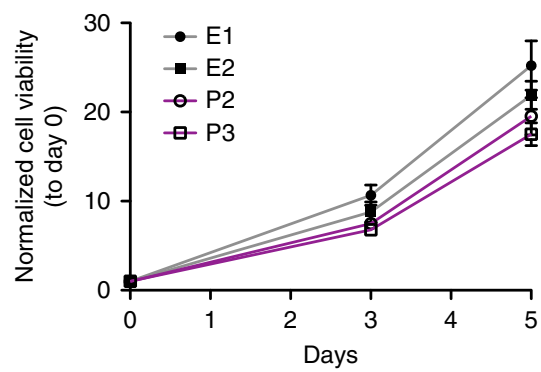

MM1402

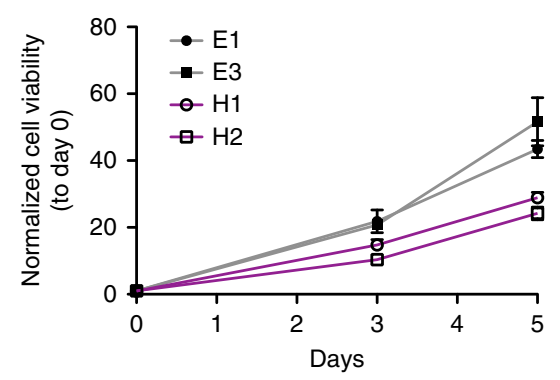

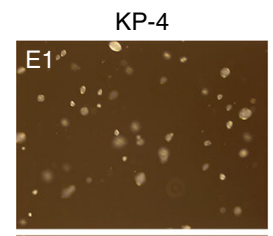

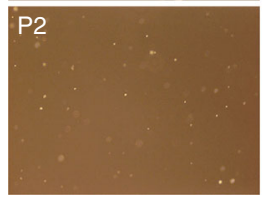

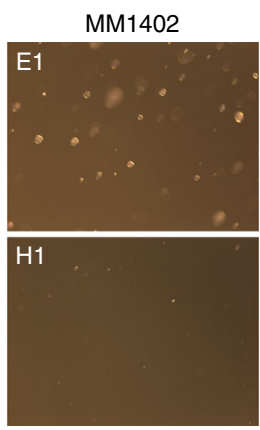

Fig. 2 KRAS is dispensable in a subset of PDAC cell lines. a Western blot confirmed loss of KRAS protein in knockout clones derived from PANC-1 (P2 complete, P3 partial), KP-4 (P1, P2, P3, P4), and MM1402 (H1, H2) cell lines compared to intact clones (PANC-1-E1,E2; KP-4-E1,E2; MM1402-E1,E2,E3). HSP90 is loading control. $\mathbf{b}$ KRAS deficient clones (purple) exhibited altered cell morphology compared to intact cells (gray). Specific differences include increased cell size, cytoplasmic translucency, and smooth edges. Scale bar is $100 \mu \mathrm{m}$. c KRAS deficient clones showed diminished proliferation in vitro. Average cell viability (normalized to day 0) \pm s.e.m. for each clone is plotted. PANC-1 knockout clone (P2) and partial knockout clone (P3) exhibited a dose-dependent effect of KRAS expression on proliferation compared to intact clones (E1, E2). d PANC-1, KP-4, and MM1402 KRAS deficient clones showed diminished soft agar colony formation. PANC-1 cells displayed a dose-dependent effect of KRAS expression on anchorage-independent growth. Scale bar is $500 \mu \mathrm{m}$ 
(Supplementary Fig. 3a, b). Furthermore, NRAS and HRAS expression were unaltered, consistent with KRAS specificity of the sgRNAs (Supplementary Fig. 3c). Mutation analysis of RNAsequencing (RNA-Seq) from 8988T (Supplementary Data 1) and A13 (Supplementary Data 2) subclones did not reveal recurrent protein-coding single nucleotide polymorphisms in expressed genes distinguishing all intact and deficient clones. Finally, we reexpressed oncogenic KRAS in deficient clones (Supplementary Fig. 3d), and observed a reversal in cell morphology (Supplementary Fig. 3e), in vitro proliferation (Supplementary Fig. 3f), and soft agar colony formation (Supplementary Fig. 3g). Together, these data demonstrate that KRAS knockout a

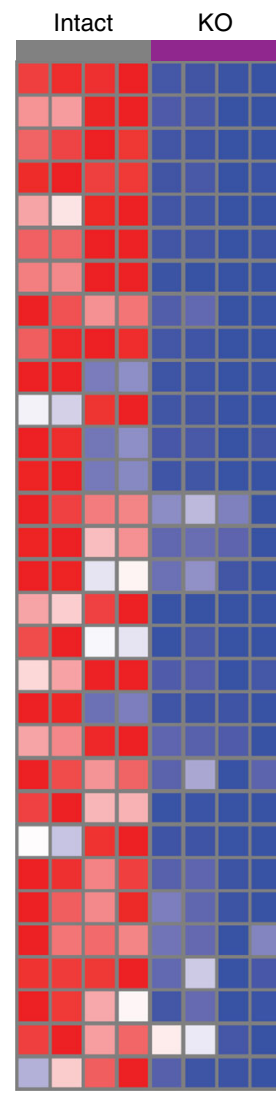

GSK2126458

BEZ235 (NVP-BEZ235)

Torin 2

MK-1775

AZD8055

Torin 1

INK 128

Elesclomol

CAY10626

BI 2536

Dasatinib (BMS-354825)

Bl6727 (Volasertib)

GSK461364

MG-132

PD 166285

17-DMAG HCl (Alvespimycin)

$\mathrm{PI}-103$

Mitoxantrone Hydrochloride

ZSTK474

Vincristine

GDC-0941

Daunorubicin $\mathrm{HCl}$ (Daunomycin $\mathrm{HCl}$ )

MLN9708

Flavopiridol hydrochloride

SB 743921

Panobinostat

Obatoclax mesylate (GX15-070)

Trichostatin A (TSA)

Epirubicin Hydrochloride

Dacomitinib (PF299804, PF-00299804)

SNS-032 (BMS-387032)

Relative
Row min

d

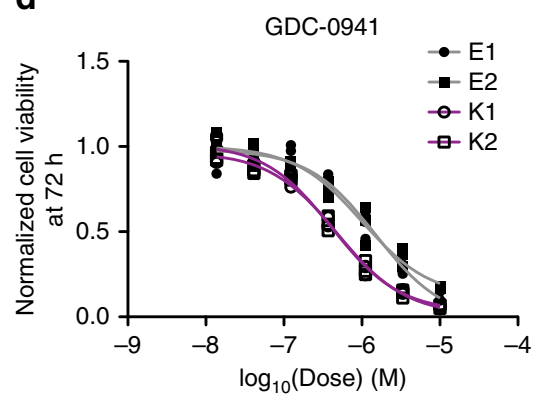

b

$\mathrm{PI} 3 \mathrm{~K} / \mathrm{mTOR}$

$\mathrm{PI} 3 \mathrm{~K} / \mathrm{mTOR}$

mTOR

mTOR

mTOR

mTOR

$\mathrm{PI3K} / \mathrm{mTOR}$

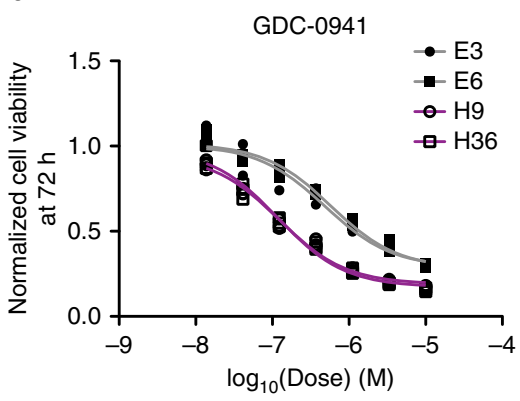

PI3K

PI3K

PI3K
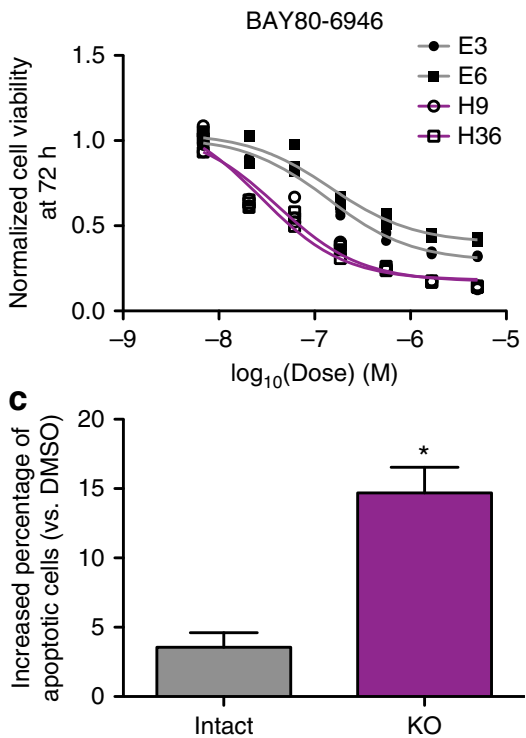

Row max

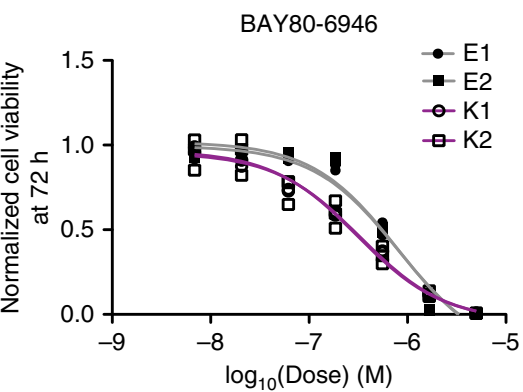

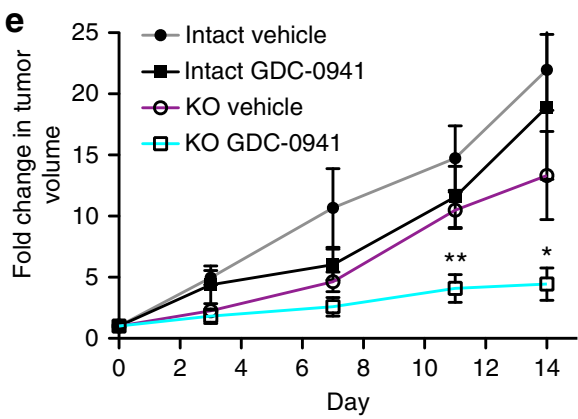

Fig. 3 KRAS deficient cells are dependent on PI3K. a Heat map of area under the curve (AUC) for KRAS intact and deficient (KO) clones (columns) treated with various compounds. Row normalized data are presented with red designating high AUC (less sensitive) and blue denoting low AUC (more sensitive). Shown are hit compounds (see "Methods" section) exhibiting greater sensitivity in KRAS deficient cells listed in order of $\triangle A U C$ from highest to lowest. PI3K and mTOR inhibitors are noted. See Supplementary Data 4 for full data set. b Dose-response curves of 8988T KRAS intact (gray) and deficient (purple) cells to the pan-PI3K inhibitors GDC-0941 and BAY80-6946. Each replicate ( $n=3$ for each dose) and curve fit are shown. c Increased apoptosis (change in percentage Annexin V-positive cells vs. DMSO) in KRAS deficient (KO) cells $48 \mathrm{~h}$ after $2 \mu \mathrm{M}$ GDC-0941 treatment. Average \pm s.e.m. is plotted ( $n=2$ clones per group). ${ }^{\star} p<0.05$, two-tailed Student's $t$ test. $\mathbf{d}$ Dose-response curves of A13 cells to pan-PI3K inhibitors. Each replicate ( $n=3$ for each dose) and curve fit are shown. e GDC-0941 significantly decreased the growth rate of KRAS deficient (KO) but not intact A13 transplanted tumors in nude mice. Average tumor volume fold increase (normalized to start of treatment at day 0$) \pm$ s.e.m. $\left(n=8\right.$ tumors per group) is plotted. ${ }^{\star} p<0.05,{ }^{\star \star} p<0.01$, two-tailed Student's $t$ test for measurements at each time point comparing GDC-0941 to vehicle 
a
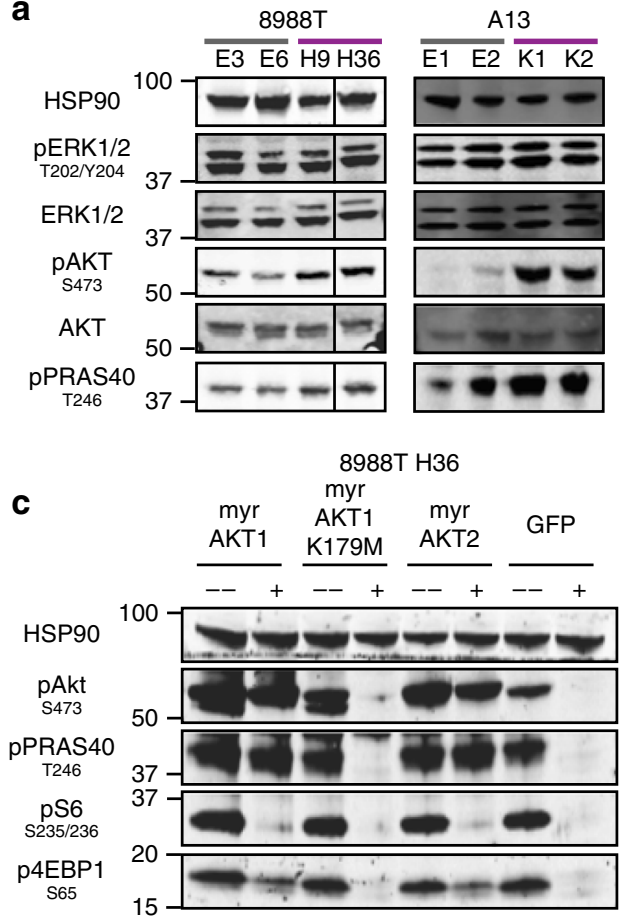

b
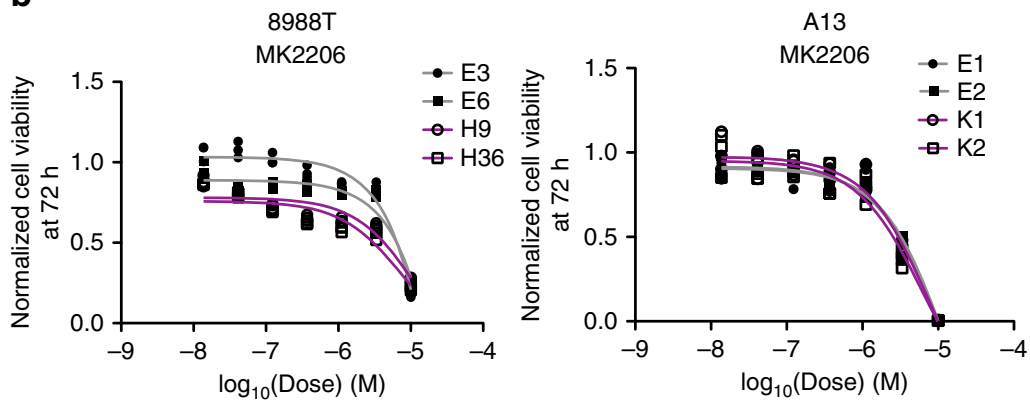

d

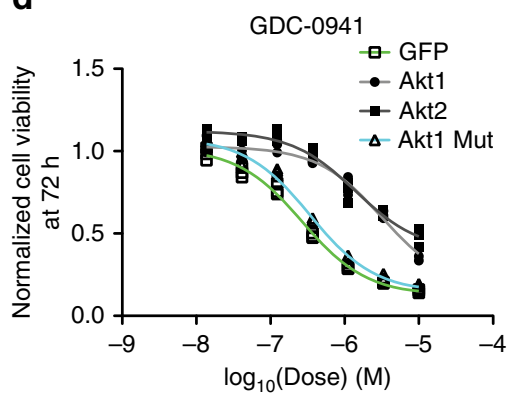

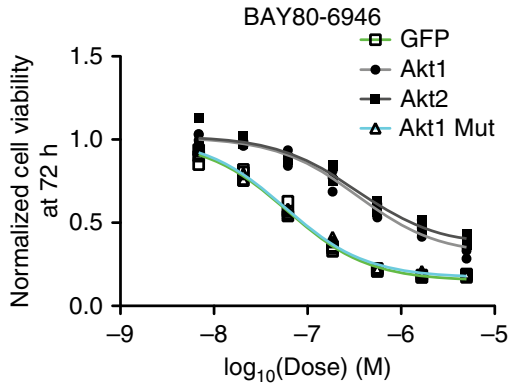

Fig. 4 PI3K inhibition functions through AKT-dependent and -independent mechanisms. a Western blot showed stable pERK1/2 but increased pAKT and pPRAS40 levels in 8988T and A13 KRAS deficient (purple) cells, consistent with PI3K/AKT pathway activation. HSP90 is loading control. b Dose-response curves of $8988 \mathrm{~T}$ and A13 KRAS intact (gray) and deficient (purples) clones to the pan-AKT inhibitor MK2206. Each replicate ( $n=3$ for each dose) and curve fit are shown. c Western blot showed sustained phosphorylation of AKT and downstream targets (PRAS40, S6, and 4EBP1) only in myr-AKT1- and myr-AKT2-expressing cells but not in myr-AKT1 (K179M)- or control GFP-expressing cells following $4 \mathrm{~h}$ of $2 \mu \mathrm{M}$ GDC-0941 treatment. d Dose-response curves of cell lines in c treated with GDC-0941 and BAY80-6946 demonstrated a marked decrease in PI3K sensitivity with myr-AKT1 or myr-AKT2 overexpression. Each replicate ( $n=3$ for each dose) and curve fit are shown

significantly impacts proliferation in vitro and tumorigenic growth in vivo, confirming the importance of KRAS in PDAC cell maintenance.

KRAS is dispensable in a subset of PDAC cells. We next evaluated the frequency of KRAS independence in a larger panel of KRAS mutant hPDAC and mPDAC cell lines derived from multiple models of KRAS-driven murine PDAC (Supplementary Table 2). We successfully isolated KRAS deficient clones from the hPDAC cell lines PANC- 1 and KP-4 and the mPDAC cell line MM1402 (Fig. 2a). These additional KRAS deficient cells exhibited cellular features comparable to those observed in 8988T and A13 knockout cells, including alterations in cell morphology (Fig. 2b), decreased proliferation in vitro (Fig. 2c), and diminished anchorage-independent growth (Fig. 2d). In contrast, we were unable to derive KRAS deficient clones from hPDAC cell lines 8902, YAPC, and PSN-1 and mPDAC cell lines D8, E, F, and MM1404, as these cell lines either generated clones that retained KRAS protein (e.g., 8902 and PSN-1, Supplementary Fig. 4a) or did not form recoverable clones at all (e.g., YAPC).

Interestingly, cell lines with lower KRAS protein levels exhibited greater tolerance for KRAS knockout (Supplementary Fig. 4b). Recent work has suggested that increased target copy number can permit CRISPR/Cas-mediated lethality in a geneindependent fashion ${ }^{25}$. Indeed, we observed that increased KRAS copy number correlated with decreased capacity to generate knockout clones, especially in hPDAC cell lines (Supplementary Fig. 4c). Nonetheless, KRAS deficient cells could be isolated from some cell lines (e.g., KP-4 and PANC-1) despite having similarly elevated KRAS copy number to cell lines that did not generate knockout clones (e.g., 8902). Together, these data are consistent with prior work ${ }^{20}$ and suggest that KRAS protein levels may be a biomarker of sensitivity to KRAS inhibition in PDAC cells.

Overall, half (3/6) of established hPDAC and one-third (2/6) of primary and established mPDAC cell lines had the capacity to generate KRAS deficient clones (Supplementary Table 3), suggesting absolute KRAS independence is not an isolated phenomenon. Moreover, we cannot exclude the possibility that screening additional clones could identify KRAS deficient cells even in parental cell lines from which we have been unable to recover knockout clones to date. Nonetheless, endogenous KRAS is dispensable in a large fraction of PDAC cells, underscoring the potential for resistance to even the very best KRAS inhibitors.

KRAS knockout cells exhibit PI3K dependence. We next sought to elucidate the mechanisms that permit the proliferation and survival of PDAC cells in the absence of KRAS. While previous work reported that marked overexpression of YAP1 could support the growth of a subset of PDAC tumors following loss of KRAS expression ${ }^{22,26}$, we did not observe similar elevations in YAP1 protein levels in KRAS deficient compared to intact cells (Supplementary Fig. 5a). Moreover, KRAS deficient cells did not exhibit increased sensitivity to verteporfin, a YAP-TEAD interaction inhibitor ${ }^{27}$, or to YAP1 knockdown/knockout compared to intact cells (Supplementary Fig. 5b-f). Therefore, we employed high-throughput drug screening to identify unique dependencies in KRAS deficient cells. We screened 8988T KRAS intact and deficient clones against a compound library comprised of kinase inhibitors, epigenetic modifiers, and chemotherapeutic agents, many of which are being tested in clinical trials or are FDA approved (Supplementary Data 3). While no compound selectively impaired the viability of KRAS intact cells, deficient 
a

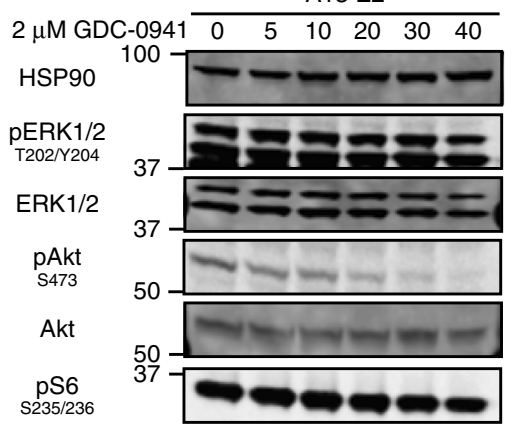

C

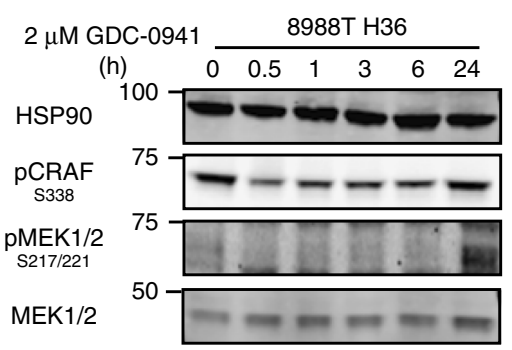

d

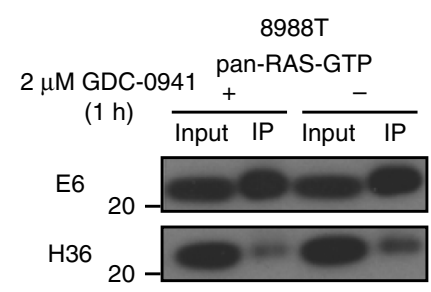

8988 T E3

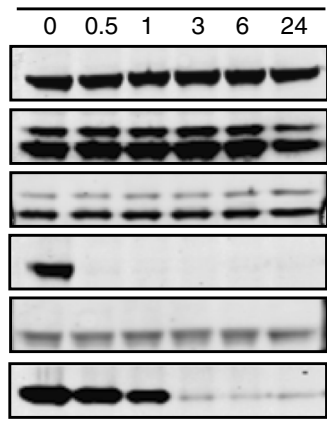

e

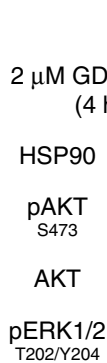

ERK $1 / 2$

$\underset{\mathrm{pS} 6}{\mathrm{~s} 235 / 236}$

S6

$\underset{\mathrm{S} 65}{\mathrm{P} 4 \mathrm{EBP} 1}$ b
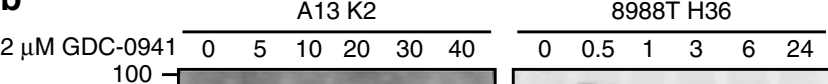

HSP90

pERK $1 / 2$

T202/Y204

ERK1/2

pAkt

Akt

$\underset{\text { p } 235 / 236}{\operatorname{An} 6}$
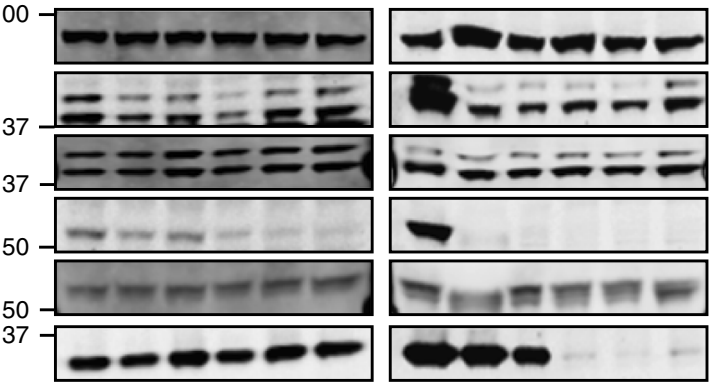

GDC-0941

f
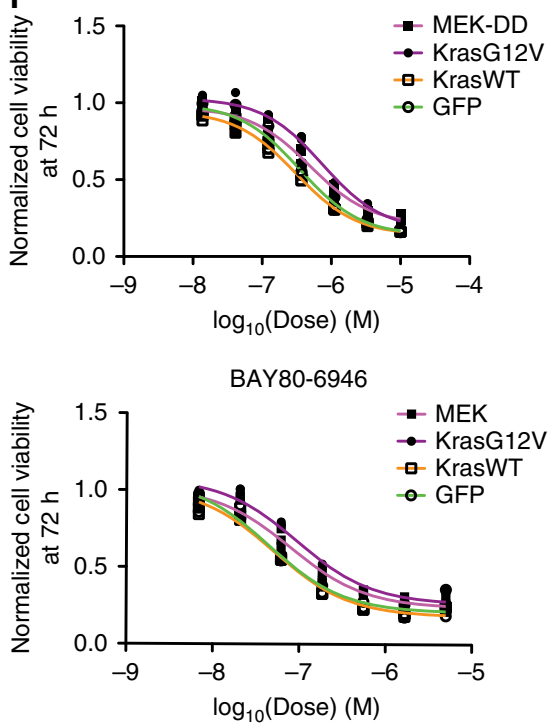

Fig. 5 MAPK blockade following PI3K inhibition in KRAS deficient cells. a Western blot showed no change in pERK1/2 levels in KRAS intact cells at designated times (minutes for A13, hours for 8988T) following GDC-0941 treatment. HSP90 is loading control. b Western blot demonstrated a transient decrease in pERK1/2 levels in KRAS deficient cells at designated times (minutes for A13, hours for 8988T) following GDC-0941 treatment. c Western blot showed a transient decrease in phosphorylation of the MAPK pathway regulators CRAF and MEK1/2 following GDC-0941 treatment in KRAS deficient cells. d Western blot of RAS-GTP levels in KRAS intact (E6) and deficient (H36) clones following 1-h treatment with GDC-0941 showed a specific decline in deficient cells. e Overexpression of constitutively active MEK (MEK-DD) or oncogenic KRAS-G12V, but not KRAS-WT or GFP, blocked pERK1/2 inhibition by a 4-h treatment with GDC-0941. f MEK-DD and KRAS-G12V-transduced cells from e showed decreased sensitivity to PI3K inhibition compared to control GFP- and KRAS-WT-transduced cells

cells exhibited increased sensitivity to pan-PI3K and mTOR inhibitors (Fig. 3a and Supplementary Data 4). As dose-response curves and direct observation suggested a cytotoxic effect of pan-PI3K inhibitors rather than the cytostatic effect of mTOR inhibition (Supplementary Fig. 6), we chose to further characterize the consequence of PI3K inhibition on KRAS deficient cells.

We confirmed enhanced sensitivity to the pan-PI3K inhibitors GDC-0941 and BAY80-6946 in additional 8988T KRAS deficient clones (Fig. 3b and Supplementary Fig. 7a) and that GDC-0941 increased apoptosis in deficient cells (Fig. 3c). In addition, we found that A13 KRAS deficient clones were more sensitive to PI3K inhibition than their intact counterparts both in vitro and in vivo (Fig. 3d, e). Finally, KP-4 and MM1402, but not PANC-1, knockout clones demonstrated increased sensitivity to GDC-0941 (Supplementary Fig. 7a). Treatment of 8988T and A13 clones with combinations of PI3K class I isoform-specific inhibitors revealed a synergistic effect of p110 $\alpha$ inhibition with p110 $\beta$ - or p1108-specific inhibitors (Supplementary Fig. 7b, c), suggesting the need for pan-class I PI3K inhibition for full effect. Biochemically, we observed stable MAPK phosphorylation but significantly increased PI3K/AKT pathway activation in 8988T and A13 deficient cells (Fig. 4a and Supplementary Fig. 7d, e). Interestingly, MM1402 and PANC-1, but not KP-4, knockout cells also showed increased pAKT levels at steady state (Supplementary Fig. 7f). These data suggest that PI3K/AKT hyperactivation and $\mathrm{PI} 3 \mathrm{~K}$ inhibitor sensitivity are features of most PDAC cells following KRAS knockout.

We next explored the mechanisms underlying PI3K/AKT activation in $8988 \mathrm{~T}$ and A13 KRAS deficient cells. As activating PI3K pathway mutations (Supplementary Data 1 and 2) and changes in the protein levels of the phosphoinositide phosphatases PTEN and INPP4B (Supplementary Fig. 8a) were not observed in deficient cells, we hypothesized that feedback stimulation of PI3K by upregulated receptor tyrosine kinases (RTKs) $)^{28-30}$ could be occurring. While we observed PDGFR $\beta$ and FGFR2 upregulation in KRAS deficient cells (Supplementary Fig. 8b), inhibition of PDGFR and FGFR alone or in combination did not show a greater effect on the proliferation of deficient cells (Supplementary Fig. 8c). Similarly, RTK array profiling of A13 intact and deficient clones did not reveal significant differences in phosphorylation across a broader array of RTKs (Supplementary 
Fig. 8d). In contrast, stimulation with any of the RTK ligands EGF, PDGF-BB, or FGF1 decreased sensitivity of deficient cells to PI3K inhibition (Supplementary Fig. 8e, f). Together, these data suggest that individual RTKs may be sufficient but not necessary to support PI3K activation in KRAS deficient cells, indicating compensatory mechanisms. Nonetheless, PI3K represents a convergent node in PDAC cells lacking KRAS function.
Simultaneous MAPK and AKT blockade by PI3K inhibition. To dissect the mechanism underlying PI3K inhibitor sensitivity in KRAS deficient cells, we evaluated the effect of the pan-AKT inhibitor MK2206 on cell viability in vitro. Surprisingly, AKT inhibition did not recapitulate the differential sensitivity observed with PI3K inhibition in $8988 \mathrm{~T}$ and A13 cells (Fig. 4b). In contrast, overexpression of constitutively active myristoylated (myr) forms

a

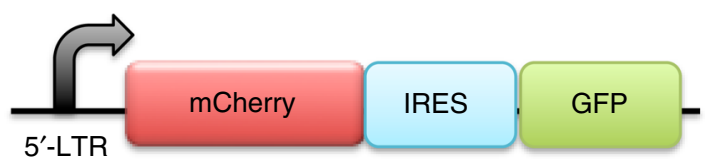

b
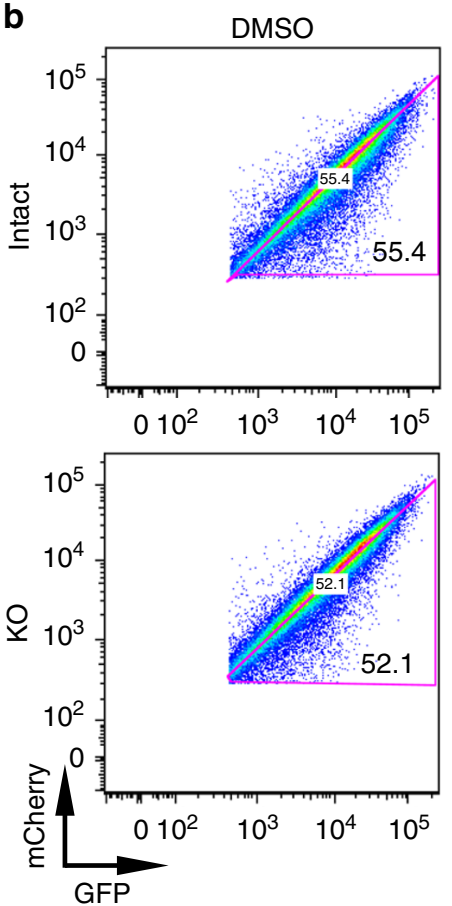

C
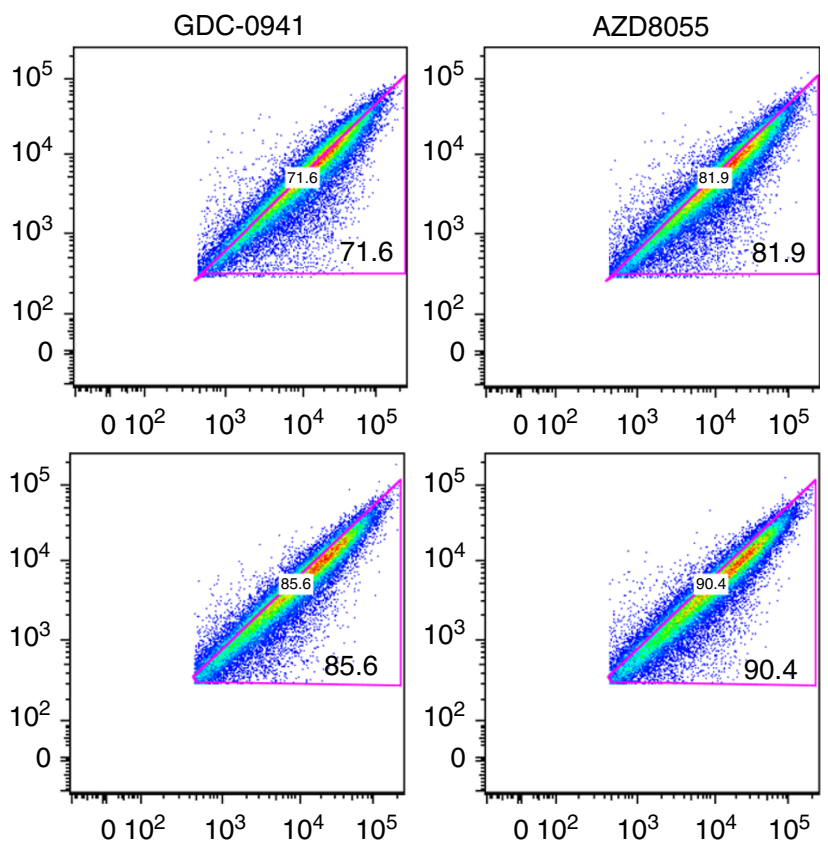
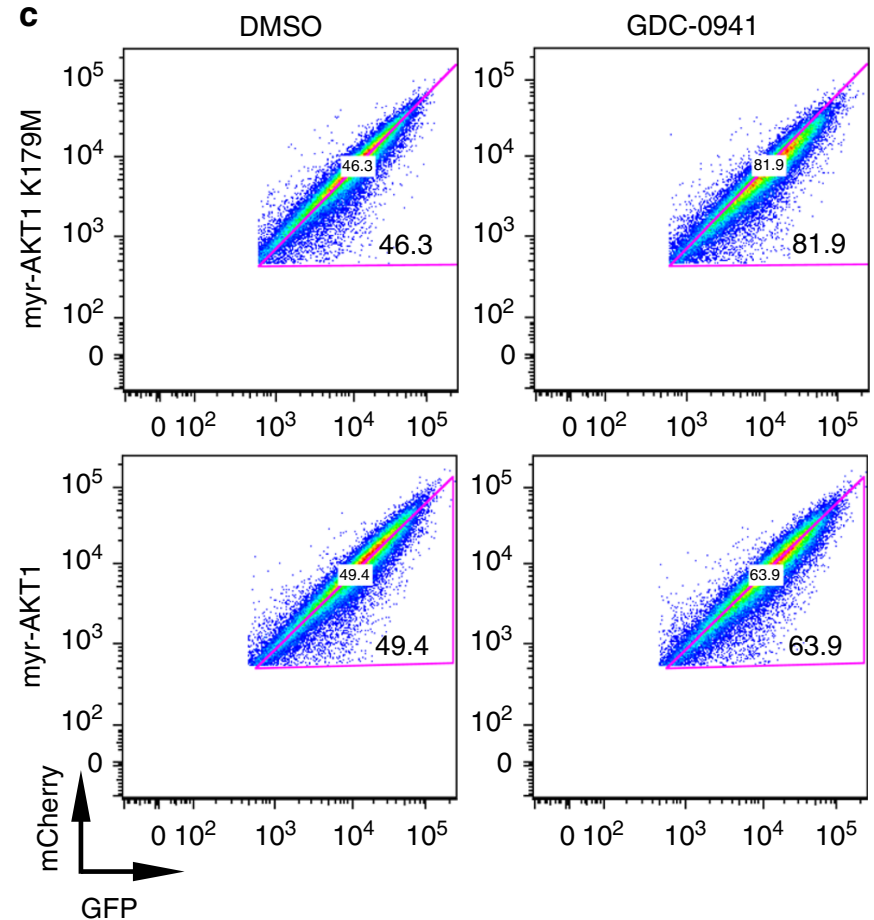
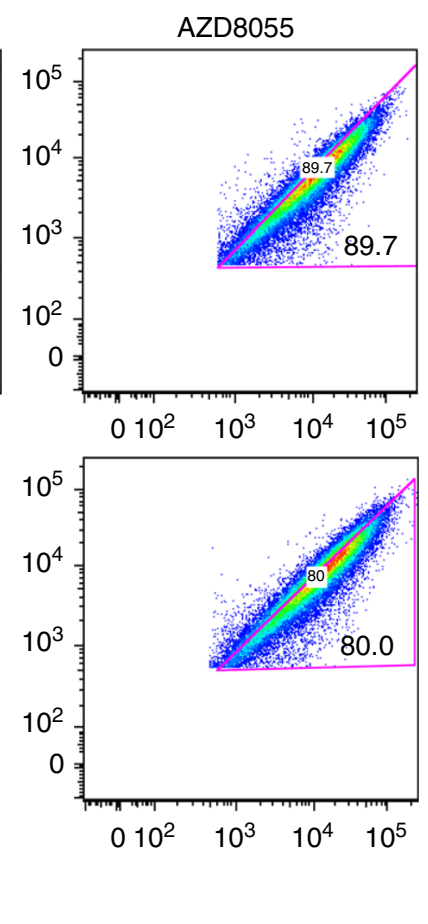
of $A K T 1$ or $A K T 2$ (but not the kinase-dead mutant AKT1 $(K 179 M))$ prevented GDC-0941-induced AKT pathway inhibition and markedly decreased PI3K inhibitor sensitivity (Fig. 4c, d and Supplementary Fig. 9a-d). Overall, PI3K inhibitor-mediated AKT blockade is necessary but insufficient for its effect on cell viability.

Recent work indicated that PI3K inhibitors inactivate the MAPK pathway in cells harboring PI3K pathway mutations to induce apoptosis 31,32 . Indeed, while GDC-0941 sustainably suppressed pAKT in both KRAS intact and deficient cells, a transient acute decrease in pERK1/2 levels lasting minutes to hours only occurred in deficient cells (Fig. 5a, b and Supplementary Fig. 10a, b). Surprisingly, the effect of GDC-0941 appeared to be due to inhibition of wild-type RAS activity upstream of the MAPK pathway, as RAS-GTP, pCRAF, and pMEK1/2 levels were diminished in KRAS deficient cells (Fig. 5c, d). Consistent with this observation, GDC-0941 also induced a decline in pERK1/2 levels in KRAS wild-type hPDAC BxPC3 and 293 human embryonic kidney cells (Supplementary Fig. 10c).

Several lines of evidence support the hypothesis that simultaneous MAPK and AKT inhibition by GDC-0941 underlie its therapeutic effect in the absence of oncogenic KRAS. First, in PANC-1 KRAS deficient cells, which do not exhibit enhanced PI3K inhibitor sensitivity, GDC-0941 effectively suppressed phosphorylation of AKT without inducing a decrease in pERK1/2 levels (Supplementary Fig. 10c). Second, the MEK inhibitor AZD6244 synergized with MK2206 in both KRAS intact and deficient $8988 \mathrm{~T}$ and A13 cells (Supplementary Fig. 10d). However, while AZD6244 enhanced the effect of GDC-0941 on KRAS intact cells, such synergy was absent in deficient cells (Supplementary Fig. 10e), likely due to the MAPK inhibitory effect already provided by GDC-0941. Finally, overexpression of constitutively active MEK (MEK-DD) or sgRNA-resistant oncogenic KRAS (but not wild-type KRAS) prevented pERK1/2 decline and reduced PI3K inhibitor sensitivity in deficient cells (Fig. 5e, $\mathrm{f}$ and Supplementary Fig. 10f, g).

Prior research has suggested that the MAPK and AKT pathways may converge on the 4EBP1-EIF4E axis to regulate cap-dependent translation in cancer cells ${ }^{33}, 34$. Interestingly, oncogenic gene signatures associated with the cap-dependent translation mediators MYC and EIF4E ${ }^{35,36}$ were enriched in gene expression analysis of 8988T KRAS intact cells (MSigDB/GSEA, Supplementary Data 11), suggesting that KRAS may also regulate this process in this cell line. While we did not observe a difference in MYC protein levels (Supplementary Fig. 8a), baseline phospho$4 \mathrm{EBP} 1$ levels were reduced in 8988T KRAS deficient cells (Supplementary Fig. 7d), permitting enhanced 4EBP1 sequestration of EIF4E to restrain cap-dependent translation. Furthermore, $K R A S$ deficient cells exhibited greater sensitivity to inhibitors of mTOR (Supplementary Fig. 6b), an important upstream regulator of translational control mediated through 4EBP1 phosphorylation. Therefore, we hypothesized that GDC-0941 treatment might further decrease 4EBP1 phosphorylation in these KRAS deficient cells, pushing them beyond a threshold to functionally impair cap-dependent translation. To test this, we transduced $8988 \mathrm{~T}$
KRAS intact and deficient cells with an mCherry-IRES-GFP translation reporter (Fig. 6a). KRAS deficient cells exhibited a more marked decrease in cap-dependent translation when treated with GDC-0941 or the mTORC1/2 inhibitor AZD8055 than intact cells (Fig. 6b). Moreover, the effects of GDC-0941 on cell viability, 4EBP1 phosphorylation, and cap-dependent translation were rescued by overexpression of myr-AKT1 but not myr-AKT1 K179M (Figs. 4c, d and 6c and Supplementary Fig. 9c, d). Taken together, cap-dependent translation may be a node of MAPK and AKT convergence, which underlies the sensitivity to PI3K inhibition in KRAS deficient cells.

Combined KRAS and PI3K inhibition as a therapeutic approach. In order to recapitulate the therapeutic effect of combined KRAS and PI3K inhibition in established PDAC tumors in vivo, we engineered A13 cells to stably express Cas9 and a DOX-inducible KRAS-targeting sgRNA (mmKras.366) and generated clones that efficiently ablated KRAS protein expression following DOX treatment in vitro (Fig. 7a). To account for clonal and animal differences, we subcutaneously transplanted two clones, one on each flank, in immunocompromised mice. Notably, there was minimal variation in tumor growth rate between the two clones. Following tumor establishment, administration of DOX feed led to mmKras.366 expression in established tumors and acute suppression of tumor growth (Fig. 7b). Importantly, KRAS inhibition alone was insufficient to maintain tumor growth suppression, possibly due to selection of escapers harboring non-frameshift mutations and/or cells that bypassed the requirement of KRAS by PI3K/AKT activation. Supporting the latter hypothesis, subsequent treatment with GDC-0941 more markedly suppressed the growth of KRAS knockout tumors (Fig. 7b).

Western blotting of tumors confirmed decreased KRAS protein levels following DOX administration (Supplementary Fig. 11a, b) and on-target inhibition of AKT phosphorylation with GDC-0941 treatment (Supplementary Fig. 11a, c). As with in vitro knockout, we saw increased phosphorylation of AKT with in vivo KRAS knockout (Supplementary Fig. 11a, c). Interestingly, we observed an increase in KRAS protein levels in DOX-treated mice also treated with GDC-0941 compared to vehicle (Supplementary Fig. 11b), suggesting selective depletion of KRAS knockout cells and outgrowth of technical escapers that did not efficiently induce loss-of-function mutations in KRAS. To evaluate for this directly, we performed massively parallel sequencing to analyze allelic fractions of the KRAS locus from these tumors (Supplementary Fig. 11d, e) with the hypothesis that there would be an increase in the proportion of non-frameshift (NFS) mutant reads (protein retained) compared to frameshift (FS) reads (protein loss) in tumors subject to combined DOX and GDC-0941 treatment. In pairwise comparisons of DOX + GDC-0941 tumors $(n=6)$ with each of the DOX tumors $(n=6)$, the NFS mutant read fraction was found to be significantly enriched in the DOX + GDC samples in $72 \%$ $(26 / 36)$ of all comparisons $\left(\chi^{2}\right.$-test of proportions, $\left.p<0.05\right)$. Collectively, these data demonstrate that PI3K inhibition

\footnotetext{
Fig. 6 PI3K inhibition enhances cap-dependent translation inhibition in KRAS deficient cells. a Schematic of cap-dependent translation reporter construct. $5^{\prime}$-LTR 5' long terminal repeat of MSCV virus with promoter activity. In transduced cells, mCherry expression correlates with cap-dependent translation and GFP expression correlates with cap-independent translation initiated via an internal ribosomal entry site (IRES). b FACS plots of GFP and mCherry fluorescence in KRAS intact and deficient (KO) cells. KRAS deficient cells exhibited a greater decrease in mCherry (relative to GFP) expression when treated for $24 \mathrm{~h}$ with GDC-0941 $(2 \mu \mathrm{M})$ or the mTORC1/2 inhibitor AZD8055 (100 nM) than intact cells. Triangle gates were drawn along the midline diagonal of the FACS plots of DMSO-treated cells and maintained in plots of drug treatment. Numbers denote percentages of cells within gate and is inversely related to cap-dependent translation of reporter. c FACS plots of GFP and mCherry fluorescence in KRAS deficient cells transduced with myr-AKT1 or myr-AKT1 (K179M). Wild-type AKT1 expression decreased the effect of GDC-0941 on cap-dependent translation compared to its kinase-dead variant
} 
a

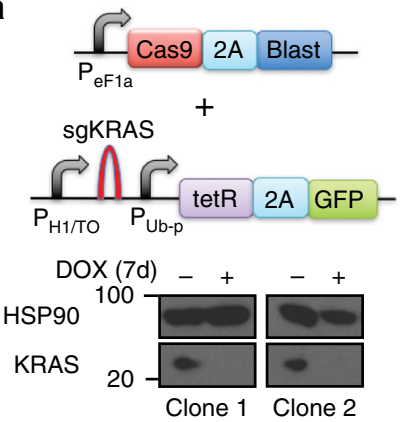

b

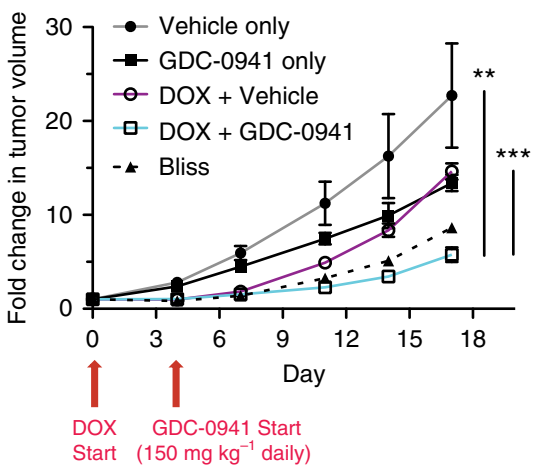

C

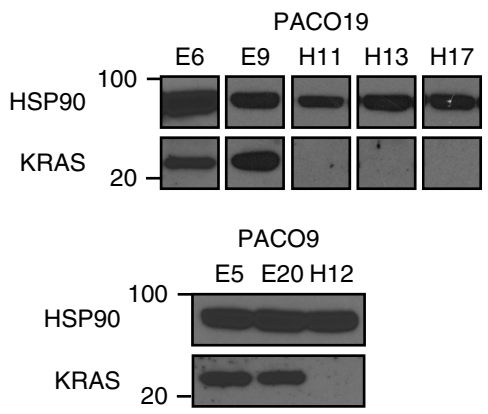

d
Reference
PACO19H11
\begin{tabular}{|llllllllllllllllllllllllllllll|}
\hline$G$ & $A$ & $T$ & $T$ & $C$ & $C$ & $T$ & $A$ & $C$ & $A$ & $G$ & $G$ & $A$ & $A$ & $G$ & $C$ & $A$ & $A$ & $G$ & $T$ & $A$ & $G$ & $T$ & $A$ & $A$ & $T$ & $T$ & $G$ & $A$ & $T$
\end{tabular}
PACO19 H13 Allele1
PACO19 H13 Allele2
PACO19H17
\begin{tabular}{llllll|lllllllllllllllllllllllllll}
$\boldsymbol{G}$ & $\boldsymbol{A}$ & $\boldsymbol{T}$ & $\boldsymbol{T}$ & $\boldsymbol{C}$ & - & - & - & - & - & $\boldsymbol{G}$ & $\boldsymbol{G}$ & $\boldsymbol{A}$ & $\boldsymbol{A}$ & $\boldsymbol{G}$ & $\boldsymbol{C}$ & $\boldsymbol{A}$ & $\boldsymbol{A}$ & $\boldsymbol{G}$ & $\boldsymbol{T}$ & $\boldsymbol{A}$ & $\boldsymbol{G}$ & $\boldsymbol{T}$ & $\boldsymbol{A}$ & $\boldsymbol{A}$ & $\boldsymbol{T}$ & $\boldsymbol{T}$ & $\boldsymbol{G}$ & $\boldsymbol{A}$ & $\boldsymbol{T}$ & -5 & $\mathrm{bp}$
\end{tabular}
PACO9H12

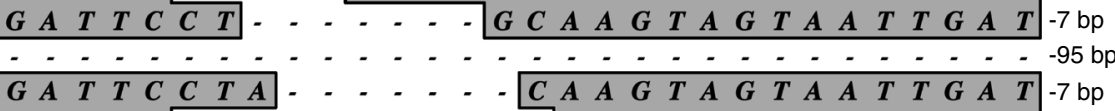
$\begin{array}{lllllllllllllllllllllllllllllllll}G & A & T & T & C & C & T & A & - & - & - & - & - & - & A & A & G & T & A & G & T & A & A & T & T & G & A & T & -7 & b p \\ G & A & T & T & C & - & - & - & - & - & - & - & - & - & - & - & A & A & G & T & A & G & T & A & A & T & T & G & A & T & -11 & b p\end{array}$

e
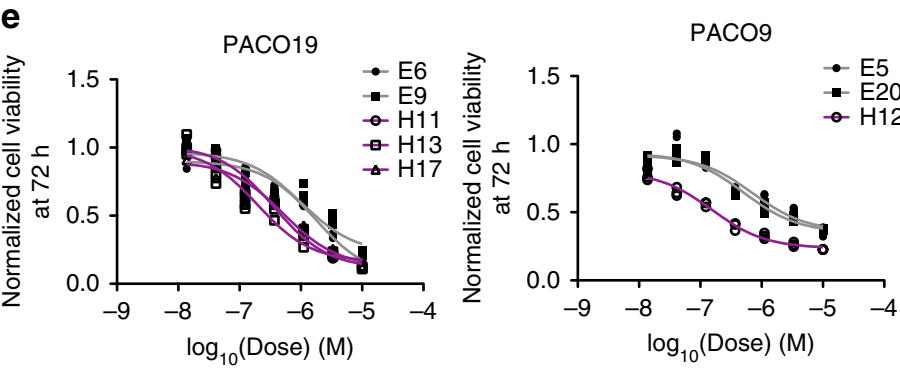

f $2 \mu \mathrm{M}$
GDC-0941 (h) pERK $1 / 2$ PERK 1204 ERK $1 / 2$ PAKT AKT

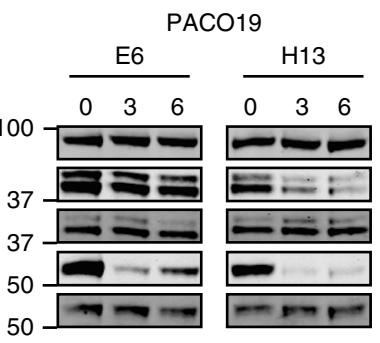

Fig. 7 Combined KRAS and PI3K inhibition as a therapeutic approach in PDAC cells. a Schematic of lentiviral constructs to express Cas 9 and a doxycycline (DOX)-inducible sgRNA targeting KRAS (sgKRAS). P efla ubiquitously expressed elongation factor 1a promoter. 2A self-cleaving peptide. Blast blasticidin resistance gene. TRE tetracycline-responsive element. $\mathrm{P}_{\mathrm{H} 1 / \mathrm{TO}} \mathrm{H} 1$ promoter with Tet-operator sites. $\mathrm{P}_{\text {Ub-P }}$ ubiquitin promoter. tetR Tet-repressor. DOX treatment relieves tetR repression of $\mathrm{H} 1$ promoter to permit sgKRAS expression. Western blot showed complete KRAS protein ablation in two different $\mathrm{A} 13$ mmKras.366 clones after 7 days of DOX treatment in vitro. b Combined KRAS (by DOX-inducible mmKras.366) and PI3K (by GDC-0941) inhibition in established subcutaneous tumors effectively inhibited tumor growth, whereas inhibition of KRAS or PI3K alone was insufficient to suppress tumor growth long term. Average tumor volume fold increase (normalized to start of DOX treatment, pooled for both clones in a) \pm s.e.m. ( $n=10$ tumors per group) is plotted. Dashed line denotes bliss independence for additive effect of DOX and GDC-0941, consistent with synergism starting at day 11. ${ }^{\star \star} p<0.01$, twotailed Student's $t$ test, DOX + GDC-0941 vs. Vehicle only at end of experiment. ${ }^{\star \star \star} p<0.001$, two-tailed Student's $t$ test, DOX + GDC-0941 vs. DOX+ Vehicle at end of experiment. c Western blot confirmed loss of KRAS protein in knockout clones derived from PACO19 (H11, H13, H17) and PACO9 (H12) compared to intact clones (PACO19-E6,E9; PACO9-E5,E20). HSP90 is loading control. d KRAS alleles from PACO19 and PACO9 clones showed out-offrame indels in KRAS deficient clones. Reference corresponds to UCSC hg19 sequence. All clones retained a single indel allele except for PACO19 H13 for which two different indels were identified, one of which was a $95 \mathrm{bp}$ deletion that is not pictured in full. The purple and orange bars denote the sgRNA and PAM sequences, respectively. e Dose-response curves of PACO19 and PACO9 KRAS intact (gray) and deficient (purple) clones to the pan-PI3K inhibitor GDC-0941. Each replicate ( $n=3$ for each dose) and curve fit are shown. $\mathbf{f}$ Western blot showed a decrease in pERK1/2 levels in KRAS deficient (H13) but not intact (E6) clones derived from PACO19 cells following GDC-0941 treatment

synergizes with acute KRAS inhibition in vivo, highlighting that this combination may be a viable therapeutic strategy in established PDAC tumors.

To further evaluate combined KRAS and PI3K inhibition as a therapeutic strategy in a clinically relevant system, we utilized CRISPR/Cas-mediated genome editing to generate KRAS deficient clones from low-passage primary patient-derived KRAS mutant hPDAC cell lines. Importantly, we were able to isolate deficient clones from both cell lines analyzed (Fig. 7c, d and Supplementary Tables 2 and 3). Similar to established hPDAC cell lines, KRAS deficient clones derived from primary hPDAC cell lines demonstrated enhanced sensitivity to PI3K inhibition (Fig. 7e) and combined MAPK and AKT blockade following pharmacological PI3K inhibition (Fig. 7f). Together, these data support the synergy of KRAS and PI3K inhibition in a variety of in vitro and in vivo PDAC models.

Identification of KRAS-regulated pathways in PDAC cells. In addition to elucidating mechanisms of resistance to KRAS inhibition, we uncovered key biological processes regulated by KRAS in PDAC cells by comparing the gene expression profiles of KRAS intact and deficient cells. Specifically, we performed RNA-Seq on multiple 8988T and A13 clones. Unsupervised hierarchical clustering cleanly segregated intact from knockout clones (Fig. 8a), and pairwise differential expression analysis identified a large number of genes with significantly altered expression (Supplementary Data 5 and 6). We performed 
independent component analysis (ICA), a blind source separation approach (see "Methods" section), to generate high-resolution gene signatures associated with KRAS knockout (Supplementary Fig. 12a, b and Supplementary Data 7 and 8). To gain insight into the knockout signature, we performed gene set enrichment analysis (GSEA) ${ }^{37,} 38$ across gene expression data sets in $\mathrm{MSigDB}^{38}$. As internal validation of our analysis, GSEA revealed anti- correlation of the knockout signatures with genes upregulated by expression of oncogenic KRAS in primary epithelial cells (Supplementary Data 9-12). We further compared our gene signatures to data sets generated using the DOX-inducible KRAS transgene mouse model to modulate KRAS levels ${ }^{22,39,40}$. Given the high degree of heterogeneity observed between tumors and conditions in these data sets, we used ICA (Supplementary Data 13) to a

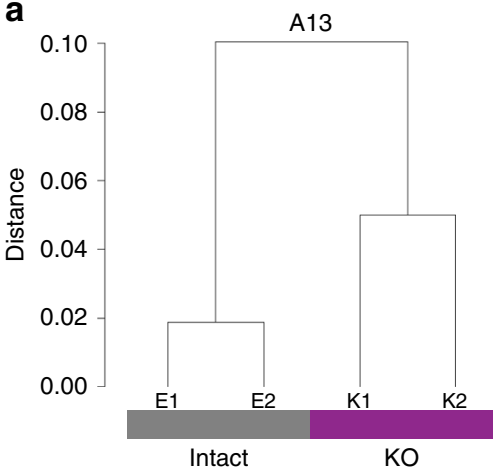

b

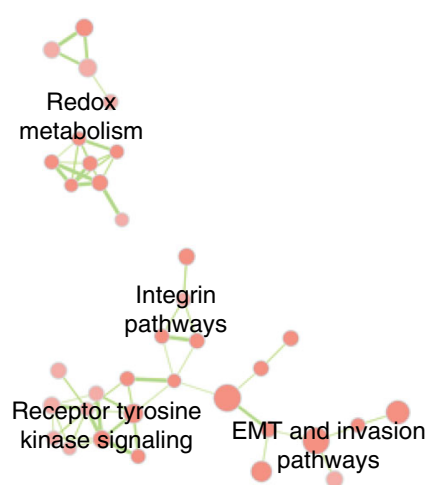

d
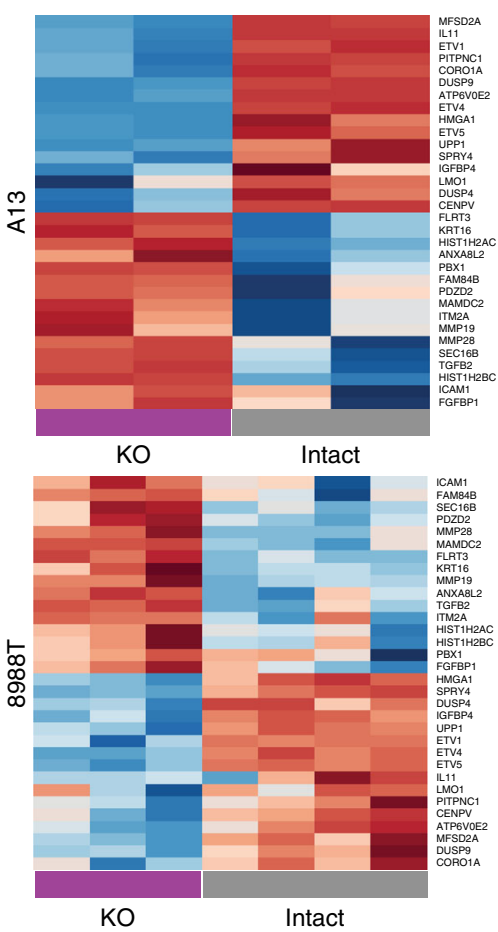

c
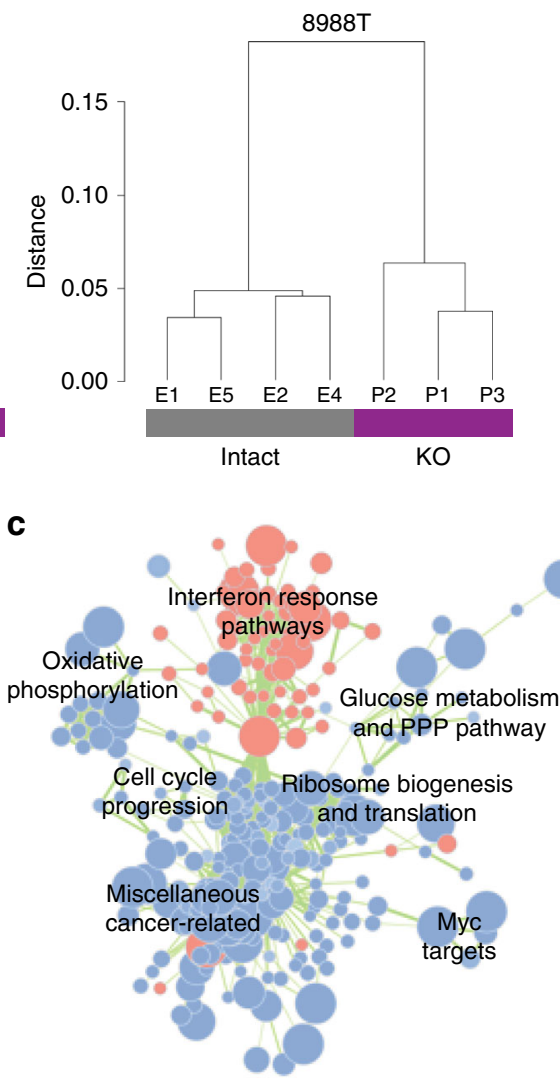

e

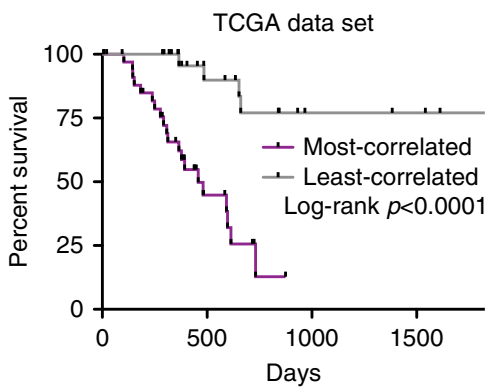

f

ICGC Data set

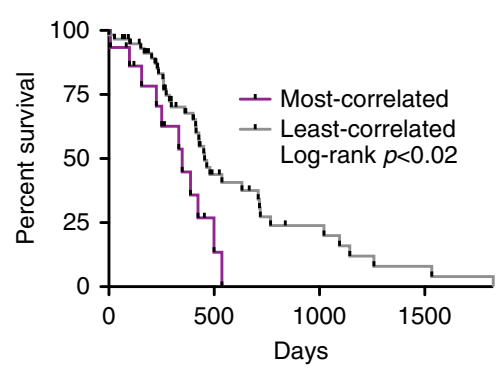


identify KRAS-ON and KRAS-OFF signatures associated with acute (24-h) KRAS withdrawal ${ }^{8}$ (Supplementary Fig. 12c), surviving cells following longer-term KRAS withdrawal $^{13}$ (Supplementary Fig. 12d), and KRAS-independent relapsed tumors in mice (Supplementary Fig. 13a). The KRASOFF signatures from these data sets were strongly enriched in our knockout signatures (Supplementary Fig. 13b), supporting the robustness of our KRAS knockout signatures across species and model systems.

We next examined GSEA results to identify key pathways regulated by KRAS in PDAC cells (Supplementary Data 14-17). A13 knockout cells showed statistically significant enrichment of a limited number of curated gene sets in pathways previously associated with KRAS, including EMT, integrin, and receptor tyrosine-kinase (RTK) signaling, and redox metabolism ${ }^{20,41,42}$ (Fig. 8b). In contrast, $8988 \mathrm{~T}$ cells exhibited alterations in a large number of biologic processes (Fig. 8c). Consistent with cellular phenotypes, 8988T knockout cells showed decreased expression of genes related to cell cycle progression, nucleotide metabolism, and oxidative phosphorylation (Fig. 8c). Importantly, we uncovered novel gene expression changes associated with KRAS function, including alterations in the expression of genes associated with ribosomal biogenesis, protein translation, and interferon response genes.

KRAS-relevant signatures predict survival in PDAC patients. Recent data from mouse models demonstrated an inverse relationship between proliferation and metastatic capacity in $\mathrm{PDAC}^{43}$. Interestingly, we observed a similar inverse relationship in the expression of genes associated with cell proliferation (cell cycle progression, nucleotide metabolism, and protein translation) and those associated with the metastatic process (EMT, invasion, and integrin pathways) based on KRAS status (Fig. 8b, c). Moreover, our knockout signatures were strongly enriched in gene expression signatures derived from circulating tumor cells (CTCs) (compared to primary tumors) in a Kras; $p 53$ mutant PDAC model $^{44}$ (Fig. 9a, b). Therefore, we hypothesized that the KRAS knockout signatures may predict worse patient survival.

We analyzed data from The Cancer Genome Atlas (TCGA) and ranked early stage (mostly resected) primary human PDAC tumors based on gene expression correlation to the knockout signatures (Supplementary Data 18). As predicted, we observed that tumors highly correlated with either the $8988 \mathrm{~T}$ or A13 knockout signatures were associated with poor patient survival (Supplementary Fig. 14a). The tumors most correlated with the knockout signatures expressed genes associated with EMT, invasion, focal adhesion/integrin signaling, and metastasis (Supplementary Fig. 14b, c). Despite their poor prognosis, these tumors showed decreased expression of ribosomal biogenesis, protein translation, oxidative phosphorylation, and cell cycle progression genes (Supplementary Fig. 14b, d). In these survival analyses, we noticed that tumors most correlated with the 8988T or A13 knockout signatures had non-significant overlap ( $p=0.44$, hypergeometric test). In contrast, tumors least correlated with the knockout signatures were largely the same regardless of the cell line from which the signature was derived $\left(p=1.04 \times 10^{-7}\right.$, hypergeometric test). This suggested that loss of oncogenic KRAS function leads to the de-repression of genes that promote a more aggressive phenotype. Consistent with this hypothesis, the murine CTC signature exhibited enrichment of gene sets downregulated by oncogenic KRAS expression in various cell types (Supplementary Fig. 9c).

We next integrated our hPDAC and mPDAC gene expression profiles to define a core set of KRAS-regulated genes with greater prognostic value. We jointly analyzed the $8988 \mathrm{~T}$ and A13 signatures to generate a 32-gene knockout signature (see "Methods" section, Fig. 8d). While the downregulated genes in this set related to MAPK signaling (DUSP4, DUSP9, SPRY4, ETV1, ETV4, ETV5), the upregulated genes supported pathways involved in the metastatic cascade, including EMT (TGFB2, $P B X 1, F G F B P 1)$, cell adhesion (FLRT3, ICAM1), and extracellular matrix breakdown (MMP19, MMP28). Strikingly, ranking tumors by expression of just the 16 upregulated genes was sufficient to improve prediction of PDAC patient survival in the TCGA cohort in multivariable analyses (Fig. 9e, Supplementary Fig. 14e, and Supplementary Data 18). We confirmed the prognostic capability of this 16-gene signature for survival in a separate PDAC patient cohort from the International Cancer Genome Consortium (ICGC) ${ }^{45}$ (Fig. 8f and Supplementary Table 4). Collectively, these data offer an independent prognostic gene signature to predict survival in early stage patients with PDAC and implicate loss of KRAS-related transcriptional suppression as a potential mechanism toward PDAC metastasis.

Finally, we examined whether our knockout signature was associated with a particular subtype of pancreatic cancer, as defined by recent gene expression profiling studies ${ }^{21}, 45,46$. Univariate and multivariable Cox regression analyses suggested that our combined knockout signature and subtype-specific signatures, including the quasi-mesenchymal ${ }^{21}$, basal ${ }^{46}$, and squamous ${ }^{45}$ subtypes defined from three independent human PDAC cohorts, were associated with worse survival within the TCGA PDAC cohort (Fig. 10a). Consistent with these results, Kaplan-Meier survival analysis using our knockout signature mirrored those seen with the subtype-specific signatures (Fig. 10b). Interestingly, we observed statistically significant overlap between the TCGA tumors most correlated (or least correlated) with our knockout signature and tumors most correlated (or least correlated) with these subtype-specific signatures (Fig. 10c). Based on these findings, we hypothesize that the quasi-mesenchymal, basal, and squamous subtypes all define a common set of tumors with distinct biology, impact on survival, and decreased KRAS function.

\footnotetext{
Fig. 8 Multiple dysregulated cellular processes in KRAS deficient cells. a Unsupervised hierarchical clustering dendrograms of $8988 \mathrm{~T}$ and A13 clones indicated clean segregation between KRAS intact and deficient $(K O)$ cells within each cell line. $\mathbf{b}$ Network representation of overlapping enriched GSEA/ MSigDB gene sets in the A13 knockout signature $(p<0.05, \mathrm{FDR}<0.25)$. Each circle represents a gene set with circle size corresponding to gene set size and intensity corresponding to enrichment significance. Red is upregulated and blue is downregulated. Each line corresponds to minimum $50 \%$ mutual overlap with line thickness corresponding to degree of overlap. Cellular processes associated with related gene sets are listed. c Network representation of overlapping enriched GSEA/MSigDB gene sets in the 8988T knockout signature $(p<0.005, F D R<0.1)$. d Heat map of a 32-gene combined knockout signature generated through ICA analysis of A13 and 8988T gene expression data sets. Gene names are listed in rows. Row normalized gene expression values are shown where red designates relative upregulation and blue designates relative downregulation. e Kaplan-Meier plots of survival of human patients from the TCGA PDAC cohort whose tumors most correlated (top quintile) and least correlated (bottom quintile) with the combined knockout signature in $\mathbf{d}$ (UP genes only). Log-rank (Mantel-Cox) $p$ value is shown. $\mathbf{f}$ Kaplan-Meier plots of survival of human patients from the ICGC cohort whose tumors most correlated (top quintile) and least correlated (remaining tumors) with the combined knockout signature in d (UP genes only). Log-rank (Mantel-Cox) $p$ value is shown
} 
a
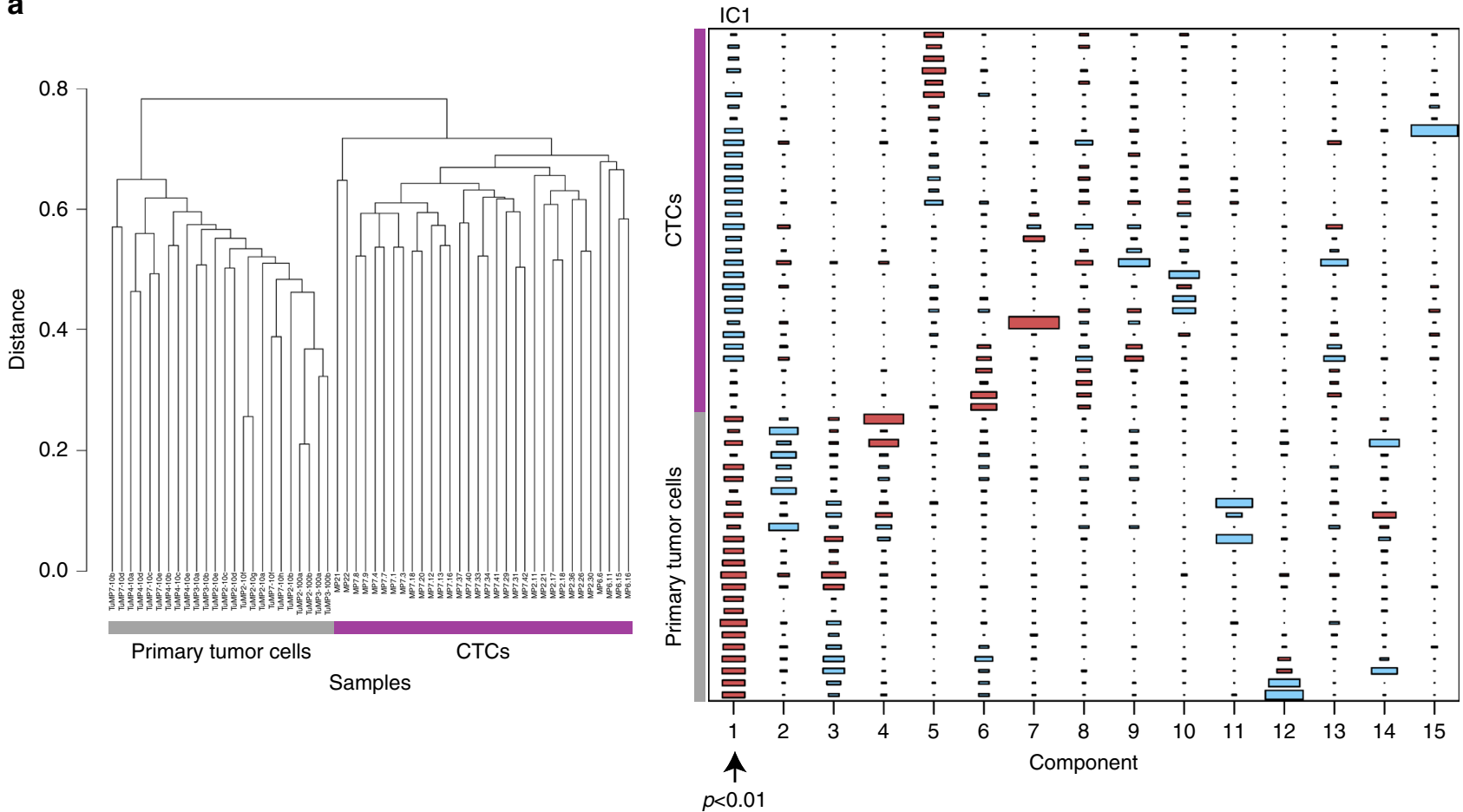

b
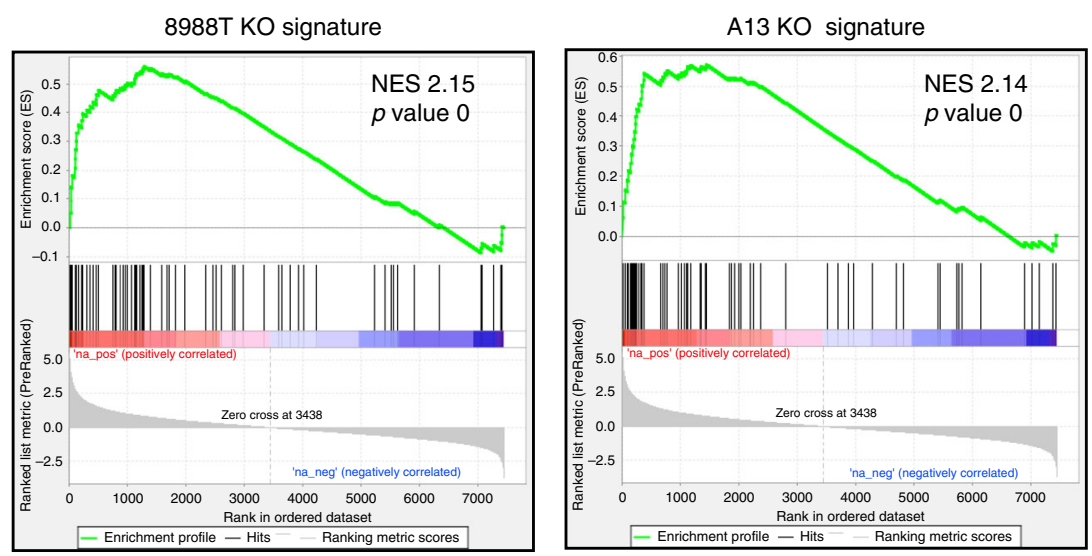

C
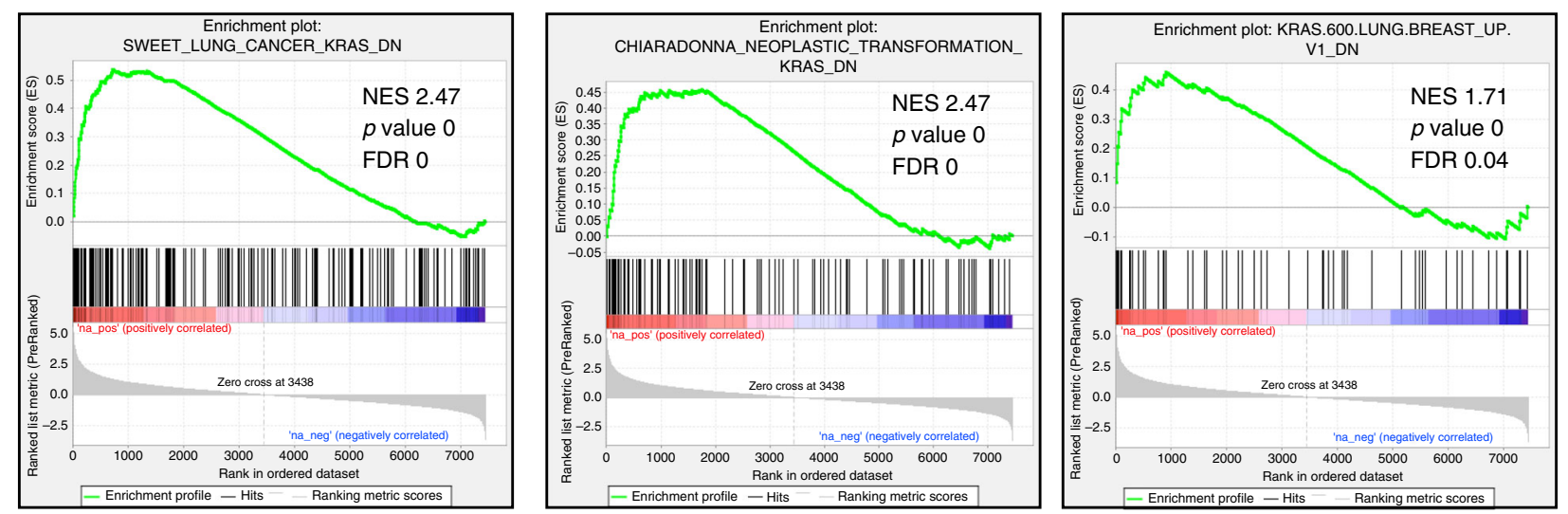

Fig. 9 KRAS knockout signatures correlate with CTC gene expression. a Unsupervised hierarchical clustering and ICA analysis of single cell RNA-Seq data from Ting et al. ${ }^{44}$ using circulating tumor cells (CTCs) and primary tumor cells from a Kras;p53 mutant mouse model. ICA analysis identified a signature (component 1 (IC1) in Hinton diagram) that distinguished CTCs from primary tumors ( $p<0.01$, Mann-Whitney U-test). b GSEA plots of 8988 T and A13 knockout signatures (top/bottom $2 \%$ genes) enriched in ICA-derived CTC signature shown above. Normalized enrichment scores (NES) and $p$ values are listed for each gene set. c Significantly enriched MSigDB gene sets in CTCs include genes downregulated (DN) following KRAS expression in the KrasLA2 lung cancer mouse model (Sweet), mouse fibroblasts (Chiaradonna), and in primary human lung and breast epithelial cells (KRAS.600). NES, $p$ values, and FDR are listed for each gene set 
a

\begin{tabular}{|c|c|c|c|c|c|}
\hline \multirow[b]{2}{*}{ Characteristic } & \multicolumn{2}{|c|}{ Univariate analysis } & \multicolumn{3}{|c|}{ Multivariable analysis } \\
\hline & $\mathrm{HR}(95 \% \mathrm{Cl})$ & $p$ value & $\mathrm{HR}(95 \% \mathrm{Cl})$ & $p$ value & $\mathrm{P}_{\text {interaction }}$ \\
\hline \multicolumn{6}{|l|}{ Clinical covariates } \\
\hline Gender (Male vs. Female) & $0.88(0.57-1.35)$ & 0.56209 & $1.11(0.66-1.87)$ & 0.6834 & \\
\hline Age (Years) & $1.03(1.01-1.05)$ & 0.00722 & $1.06(1.03-1.09)$ & 3.83E-05 & 0.09937 \\
\hline T score (T3 vs. T1/T2) & $2.51(1.24-5.08)$ & 0.01005 & $1.29(0.56-2.99)$ & 0.54968 & \\
\hline N score (N1 vs. N0) & $2.16(1.24-3.75)$ & 0.00647 & $0.77(0.34-1.73)$ & 0.53009 & \\
\hline Completeness of resection (R1 vs. R0) & $1.95(1.23-3.09)$ & 0.00461 & $1.82(1.03-3.21)$ & 0.03817 & 0.7284 \\
\hline Number of lymph nodes & $1.06(1.01-1.12)$ & 0.02096 & $1.15(1.03-1.27)$ & 0.00898 & 0.5739 \\
\hline \multicolumn{6}{|l|}{ Signatures (standardized scores) } \\
\hline KRAS KO (combined) & $1.94(1.46-2.57)$ & $4.74 \mathrm{E}-06$ & $2.03(1.04-3.98)$ & 0.03929 & - \\
\hline Collisson classical & $1.64(1.18-2.27)$ & 0.00295 & $1.52(0.48-4.88)$ & 0.47718 & \\
\hline Collisson exocrine & $1.07(0.86-1.33)$ & 0.54559 & $0.64(0.32-1.30)$ & 0.21961 & \\
\hline Collisson QM & $2.08(1.53-2.82)$ & $3.02 \mathrm{E}-06$ & $3.16(1.45-6.88)$ & 0.00374 & 0.06652 \\
\hline Moffitt basal & $1.80(1.40-2.30)$ & $3.51 \mathrm{E}-06$ & $0.72(0.33-1.56)$ & 0.40155 & \\
\hline Bailey ADEX & $0.88(0.69-1.11)$ & 0.27263 & $1.57(0.73-3.41)$ & 0.25076 & \\
\hline Bailey immunogenic & $1.00(0.82-1.22)$ & 0.98072 & $0.79(0.54-1.16)$ & 0.22939 & \\
\hline Bailey progenitor & $1.22(0.97-1.53)$ & 0.08414 & $0.95(0.44-2.03)$ & 0.89605 & \\
\hline Bailey squamous & $1.65(1.32-2.07)$ & 0.00001 & $0.65(0.24-1.76)$ & 0.39675 & \\
\hline
\end{tabular}

HR=Hazard ratio; $\mathrm{Cl}=$ Confidence interval; $T$ score=primary tumor size/invasiveness; $N$ score=Lymph node metastasis

$P_{\text {interaction }}=p$ value of interaction between significant covariates (likelihood ratio test)

b

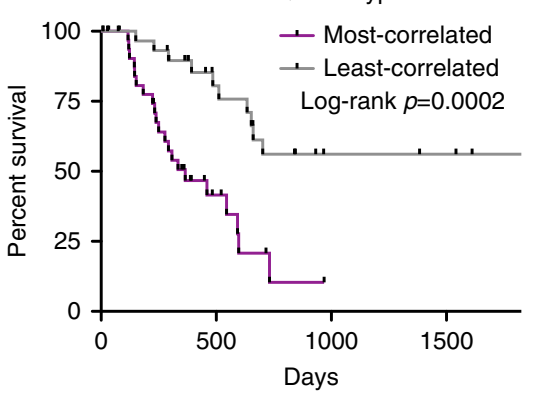

Moffitt Basal subtype

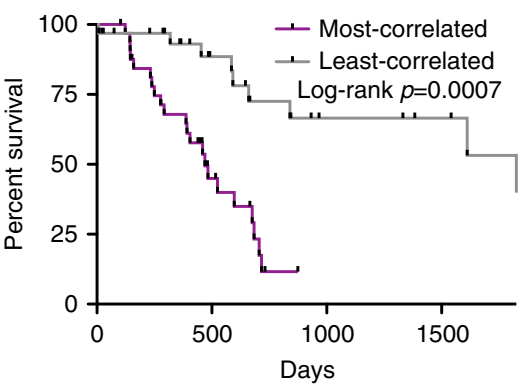

Bailey squamous subtype

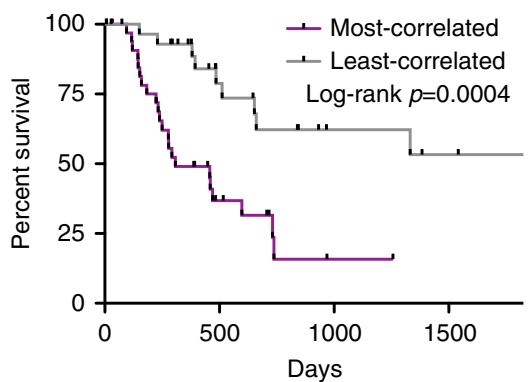

C

Most-correlated tumors/patients

\begin{tabular}{|c|c|c|c|c|}
\hline Tumor group & KRAS KO & Collisson & Moffitt & Bailey \\
\hline KRAS KO & & 51.5 & 57.6 & 48.5 \\
\hline Collisson & $3.40 \mathrm{E}-06$ & & 48.5 & 69.7 \\
\hline Moffitt & $3.91 \mathrm{E}-08$ & $2.40 \mathrm{E}-05$ & & 66.7 \\
\hline Bailey & $2.40 \mathrm{E}-05$ & $4.63 \mathrm{E}-13$ & $1.09 \mathrm{E}-11$ & \\
\hline
\end{tabular}

Least-correlated tumors/patients

\begin{tabular}{|c|c|c|c|c|}
\hline Tumor group & KRAS KO & Collisson & Moffitt & Bailey \\
\hline KRAS KO & & 60.6 & 69.7 & 66.7 \\
\hline Collisson & $3.14 \mathrm{E}-09$ & & 60.6 & 75.8 \\
\hline Moffitt & $4.63 \mathrm{E}-13$ & $3.14 \mathrm{E}-09$ & & 69.7 \\
\hline Bailey & $1.09 \mathrm{E}-11$ & $3.98 \mathrm{E}-16$ & $4.63 \mathrm{E}-13$ & \\
\hline
\end{tabular}

Fig. 10 KRAS knockout signature overlaps with subtype-specific signatures of PDAC. a Univariate and multivariable Cox proportional hazards models on overall survival in the entire TCGA PDAC cohort, including clinical covariates and gene expression signatures, revealed that the combined KRAS knockout signature was independently associated with worse survival. Hazard ratios (HR) and $p$ values (Cox regression) are reported. A comparison between a model with and without an interaction term (likelihood ratio test) was used to determine independence $(p>0.05)$ between significant covariates in multivariable analysis and the combined KRAS knockout signature. b Kaplan-Meier plots of survival in human PDAC patients from the TCGA cohort based on tumors most correlated (top quintile) and least correlated (bottom quintile) with the quasi-mesenchymal (QM) subtype from Collisson et al. ${ }^{21}$, basal subtype signature from Moffitt et al. ${ }^{46}$, and the squamous subtype signature from Bailey et al. ${ }^{45}(n=33$ most correlated and $n=33$ least correlated tumors). Log-rank (Mantel-Cox) $p$ values are shown. c Tables show percentage of tumor/patient overlap of top (most correlated) and bottom (least correlated) quintiles of tumors (out of $n=33$ per group) in each signature. Green shading corresponds to degree of overlap ( white $=0 \%$, dark green $=$ $100 \%$ ). $p$ values for significance of overlap (between $n=33$ groups) within the TCGA PDAC cohort (out of 166 total tumors) are also shown (hypergeometric test). Red shading corresponds to significance of overlap (white $=0.05$, dark red $=$ minimum $p$ value)

\section{Discussion}

Here we investigated the consequence of complete endogenous $K R A S$ ablation in PDAC cells. By inducing loss-of-function mutations in KRAS, we modeled the cellular effects of complete inhibition to predict resistance mechanisms to KRAS inhibition and to uncover KRAS-regulated pathways in PDAC. Given the significant adverse effects of KRAS knockout on in vitro proliferation and in vivo tumorigenic growth even in knockouttolerant cells, our study adds to prior work supporting the merit of KRAS-directed therapies for PDAC ${ }^{10,18}$. Furthermore, the dose-response relationship between KRAS levels and cellular phenotypes observed (e.g., PANC-1 clones in Fig. 2) highlights 
the therapeutic benefit of developing the most potent inhibitor possible. With the significant public and private investment toward the generation of novel KRAS-targeted $\operatorname{drugs}^{17}$, 18, elucidation of potential resistance mechanisms concurrent with the development of these inhibitors will facilitate their effectiveness in the clinic.

Recent studies have implicated YAP1 overexpression as a means to escape KRAS inhibition in a subset of tumors and cell lines $^{22,} 26$. Our KRAS knockout cells revealed an alternative bypass mechanism supported by canonical (i.e., AKT pathway) and non-canonical (i.e., MAPK pathway) PI3K signaling. Given the variety of resistance mechanisms that arise in response to tyrosine-kinase inhibition in RTK-mutated cancers, it is unsurprising that multiple pathways could maintain PDAC cell survival following KRAS inhibition in various contexts. Importantly, we observed PI3K dependence in KRAS deficient clones derived from 6/7 (86\%) parental cell lines, suggesting that PI3K represents a convergent target regardless of tumor cell line or species of origin.

While PI3K signaling can contribute to PDAC development in mouse models, its role in PDAC maintenance has been less clear. Expression of oncogenic PIK3CA in the developing pancreas phenocopies pancreatic cancer initiation and progression observed in KRAS mutant mice ${ }^{47}$, 48 . In contrast, PI3K inhibition alone has only marginal benefit in preclinical mouse PDAC models, whereas combination therapies with MEK inhibition demonstrate significantly greater anti-tumor effects ${ }^{49-51}$. In accordance, combined MAPK and AKT blockade exhibited by single-agent PI3K inhibitors in PDAC cells is only evident when oncogenic KRAS is absent. Although the MAPK pathway

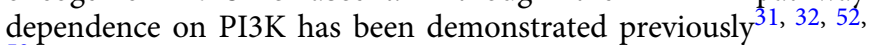
${ }^{53}$, we reveal a novel induced dependency on PI3K activity due to loss of oncogenic KRAS expression and provide evidence that this rewiring occurs at the level of wild-type RAS.

Despite the fact that PI3K has typically been considered a RAS effector, a growing body of evidence suggests that PI3K can act upstream to stimulate RAS-MAPK signaling in various contexts. Our data demonstrates that RAS mutant cancer cells in which mutant RAS is ablated or RAS WT PDAC cells (e.g., BxPC3) show PI3K-dependent MAPK activation. Recent work from Rosen and colleagues revealed that PI3K inhibition in breast cancer cells harboring oncogenic PI3K mutations induces a transient decrease in RAS-MAPK signaling, which could be blunted by expression of exogenous mutant $R A S^{32}$. Interestingly, this response is not limited to cancer cells, as PI3K inhibition in human embryonic kidney cells (Supplementary Fig. 10c) and hematopoietic progenitor cells $s^{54,55}$ also reduced MAPK signaling. Though the precise nature of how PI3K stimulates RAS-MAPK activation in KRAS deficient PDAC cells remains unclear, we speculate that this could be due to alterations in GAP activity, recruitment via Gab1/Shp2 ${ }^{56}$, or phospholipid signaling ${ }^{54}$. Nonetheless, we show that PI3K transitions to an upstream targetable regulator of not only the canonical AKT pathway, but also the RAS-MAPK pathway when oncogenic KRAS is lost. Additionally, we demonstrate that biochemical evidence of transient MAPK inhibition is a better predictive biomarker for PI3K inhibitor sensitivity than PI3K/AKT pathway hyperactivation (Supplementary Figs. 7 and 10b, c).

We further took advantage of KRAS intact and deficient cell lines to identify novel KRAS-regulated pathways in PDAC. These include the upregulation of genes associated with ribosomal biogenesis and protein translation and the downregulation of genes associated with interferon response and the metastatic cascade. Moreover, we developed a 16-gene signature, suppressed by KRAS, with independent prognostic value in PDAC validated in two different patient cohorts (TCGA and ICGC). Finally, we demonstrated that our KRAS knockout signatures are enriched in PDAC CTCs relative to primary tumors. Given these data and evidence that CTCs display expression of genes downregulated by oncogenic KRAS in multiple contexts, we propose that decreased KRAS activity may promote gene expression changes that drive metastasis. Moreover, we hypothesize that the poor prognoses observed with the quasi-mesenchymal, basal, and squamous subtypes is a consequence of decreased KRAS activity leading to greater metastatic potential in these human tumors. Consistent with this hypothesis, epigenetic rather than genetic mechanisms appear to mediate metastasis in human PDAC tumors ${ }^{57,58}$. While further work is needed in primary human specimens to confirm the relationship between KRAS activity and metastasis, our work highlights KRAS-relevant gene signatures as independent prognostic factors in PDAC.

In summary, we have shown that KRAS, the hallmark mutated gene in PDAC, is dispensable in a subset of PDAC cells. We identified novel functions of KRAS in altering gene expression balancing proliferation and metastasis. Finally, our data demonstrate that canonical and non-canonical PI3K pathway activation may bypass the requirement for KRAS in PDAC and that simultaneous inhibition of KRAS and PI3K may be a viable combinatorial therapeutic strategy for this disease.

\section{Methods}

Cell lines and culture conditions. Cell lines used in this study are described in Supplementary Table 2. Established human PDAC cell lines were obtained from the Broad Institute Cancer Cell Line Encyclopedia, sourced from DSMZ-Germany, American Type Culture Collection (ATCC), and RIKEN, and identity authenticated by DNA fingerprinting by the Broad Institute. Primary human PDAC cell lines (PACO9 and PACO19) were obtained as previously described ${ }^{59}$. Briefly, cell lines were derived from pancreatic cancer tissue specimens obtained from patients admitted to the Department of General, Visceral, and Transplantation Surgery at the University of Heidelberg under a protocol approved by the ethical committee of the University of Heidelberg (301/2001) and conducted in accordance with the Helsinki Declaration. Informed consent was obtained from all patients. Mouse PDAC cell lines were derived from primary pancreatic tumors from $L S L-K r a s{ }^{G 12 D}$; p53 flox/flox; $P d x 1$-CreER mice treated with tamoxifen (Sigma) to induce oncogenic Kras $^{G 12 D}$ activation and biallelic $p 53$ inactivation in the pancreas ${ }^{60}$ or from the LSL-Kras ${ }^{G 12 D}$; $p 53^{R 172 H / W T}$; Pdx1-Cre (KPC) mouse model ${ }^{61}$. All cell lines tested negative for mycoplasma by PCR testing.

Cells were maintained in DMEM (Corning Cellgro) supplemented with 10\% fetal bovine serum (FBS) (Hyclone) and penicillin/streptomycin or RPMI-1640 (Corning Cellgro) supplemented with 10\% FBS, L-glutamine, and penicillin/ streptomycin. PACO cell lines were grown in serum-free medium as previously described $^{59}$. For inducible-shRNA experiments, doxycycline (DOX, Sigma) was used at $1 \mu \mathrm{g} \mathrm{mL}^{-1}$ in culture media and replaced every $2-3$ days. Cell viability was analyzed after 5 days of DOX treatment using the CellTiter-Glo (CTG)

luminescence assay (Promega), which measures cellular ATP levels as a surrogate for cell number and growth. Luminescence was read on a Tecan M2000 Infinite Pro plate reader. Cells were imaged with a Nikon Eclipse TE2000-U light microscope and SPOT RT3 camera.

Cloning of sgRNA and overexpression constructs. Lentiviral constructs for CRISPR/Cas-mediated genome editing from the dual-vector lentiviral GeCKOv2 system, lentiCas9-Blast and lentiGuide-Puro ${ }^{62}$ (Supplementary Fig. 1a), were provided by Dr Feng Zhang. sgRNAs targeting human and mouse KRAS exons were designed (Supplementary Table 1) and ligated into the BsmBI site with compatible annealed oligos. lentiGuide-Hygro (Supplementary Fig. 1a) was generated following sgRNA ligation by subcloning a hygromycin resistance gene PCR amplified from MSCV-Luciferase-pPGK-Hygro (Addgene \#18782) into BsiWI and MluI sites. pLKO-Tet-On constructs (Supplementary Fig. 1c) targeting KRAS (shKRAS.407) and LACZ (shLACZ.1650) and pLKO constructs targeting YAP1 (shYAP1.1573 and shYAP1.1928) and LACZ (shLACZ.1650) were provided by Dr William Hahn (Supplementary Table 1). mmKras.366 sgRNA targeting mouse Kras was cloned into a lentiviral vector for DOX-inducible sgRNA expression provided by Dr Marco Herold ${ }^{63}$. To generate lentiviral constructs for overexpression, we first produced $L V$ - $p S V 40-m$ Cherry-pPGK-FlpO, LV-pSV40-Puro-pPGK-eGFP, and $L V$-pSV40-Blast-pPGK-eGFP by assembling four parts with overlapping DNA ends into a $5.7 \mathrm{~kb}$ lentiviral backbone using Gibson assembly (New England Biolabs) as previously described ${ }^{64}$. PCR amplified cDNAs (Supplementary Table 5) were subcloned into AfeI and AscI sites to replace FlpO and $e G F P$ and verified by sequencing and restriction digest. Alternatively, cDNAs were subcloned into the EcoRI and BamHI sites of pBABE-zeo (Addgene \#1766) or pBABE-puro (Addgene \#1764) retroviral vectors. The translation reporter construct MSCV-mCherry-IRES- 
GFP was generated by subcloning PCR amplified mCherry into the XhoI and EcoRI sites of MSCV-IRES-GFP (Addgene \#9044). High-fidelity restriction enzymes (New England Biolabs) were used for restriction digests when available. Cloning methods and primer sequences not detailed in this manuscript are available upon request.

Lentiviral and retroviral production and transduction. For lentiviral infections, lentiviral backbone, packaging vector (delta8.2 or psPAX2), and envelope (VSV-G) were transfected into 293T cells with TransIT-LT1 (Mirus Bio). Supernatant was collected at 48 and $72 \mathrm{~h}$ and applied to target cells with $8 \mu \mathrm{gL}^{-1}$ polybrene (EMD Millipore) for transduction. Transduced cells were treated with $10 \mu \mathrm{g} \mathrm{mL}^{-1}$ blasticidin S (Life Technologies), $2-4 \mu \mathrm{g} \mathrm{mL}^{-1}$ puromycin (Life Technologies), $400 \mu \mathrm{g}$ $\mathrm{mL}^{-1}$ hygromycin B (Roche), or $400 \mu \mathrm{g} \mathrm{mL}^{-1}$ zeocin (Life Technologies) for 3-7 days, as appropriate, for antibiotic selection. Alternatively, Cas9-Blastexpressing cells were transfected with lentiGuide-Puro harboring sgKRAS to induce KRAS knockout (8988T T1 and T2 cells) using the Amaxa Nucleofector Kit for Mammalian Epithelial Cells (Lonza). To generate single cell clones from sgKRAStransduced cells, we sorted one cell per well into 96-well plates using a FACSAria II (Becton Dickinson) or MoFlo (Beckman Coulter) FACS sorter. Alternatively, PACO clones were manually picked from culture plates following low-density plating. Intact clones were derived from lentiGuide-Puro- or lentiGuide-Hygrotransduced cells without inserted guide sequence (empty vector) except for $8988 \mathrm{~T}$ E1, which was derived from $h S K R A S .22 V$-transduced cells but exhibited loss of Cas9 protein expression, no mutagenesis, and KRAS intact cell properties. sgKRAS vectors used to generate knockout clones are listed in Supplementary Table 3. For overexpression constructs harboring $m$ Cherry, fluorescent cells were sorted using a FACSAria II sorter. For retroviral infections, the retroviral backbone and pCL-Eco (for mouse cells) or pUVMC and VSV-G (for human cells) were transfected into 293T cells. For the translational reporter experiments, fluorescence was assessed using an LSR II FACS analyzer (Becton Dickinson) and data were analyzed using FlowJo software.

In vitro growth assays. Anchorage-independent growth was assessed by plating 10,000 cells in $0.4 \%$ low melting temperature agarose (Seaplaque) in complete media on top of a $0.8 \%$ preformed agarose layer. Cells were grown for 9-14 days and colonies were stained with $0.5 \%$ crystal violet and destained with water. Microscopic images of the colonies were taken pre- and post-crystal violet staining. For growth curves, 250-1000 cells were plated on day 0 and grown for 5 days in culture. Four-five replicates for each cell line per day were assessed for cell viability by CTG assay. Cell viability results were normalized to luminescence at day 0 . Three-dimensional culture was established by plating $250-500$ cells from a single cell suspension onto a growth factor-reduced matrigel (Corning) layer, allowing cell migration into matrigel for $4-6 \mathrm{~h}$. Cells were grown in complete media for 7-10 days prior to analysis. Three technical replicates were performed for each clone. Apoptosis was measured using the Guava Nexin Reagent per the manufacturer's instructions and analyzed on a Guava flow cytometry system (Millipore).

Immunoblotting. Cells were lysed with ice-cold RIPA buffer (Pierce), supplemented with $0.5 \mu \mathrm{M}$ EDTA and Halt protease and phosphatase inhibitors (Thermo Scientific), rotated at $4{ }^{\circ} \mathrm{C}$ for $15-30 \mathrm{~min}$ to $\mathrm{mix}$, and centrifuged at maximum speed for $15 \mathrm{~min}$ to collect whole cell lysates. Protein concentration was measured with the BCA protein assay (Pierce). An aliquot of $20-30 \mu \mathrm{g}$ of total protein per sample was loaded into $4-12 \%$ Bis-Tris gradient gels (Life Technologies) and separated by SDS-PAGE. Proteins were transferred to nitrocellulose (for LI-COR) or PVDF (for ECL) membranes. The following antibodies were used for immunoblotting: mouse anti-HSP90 (BD \#610418, 1:10,000), rabbit anti-beta-tubulin (CST 2128, 1:1000), mouse anti-KRAS (SCBT sc-30, 1:200), mouse anti-NRAS (SCBT sc-31, 1:200), mouse anti-HRAS (SCBT sc-29, 1:200), rabbit anti-pERK1/2 (T202/Y204) (CST 4370, 1:1000), mouse anti-ERK1/2 (CST 9107, 1:1000), rabbit anti-pAKT(S473) (CST 4060, 1:2000), rabbit anti-pAKT(T308) (CST 2965, 1:1000), mouse anti-AKT (CST 2966, 1:2000), rabbit anti-pPRAS40(T246) (CST 2997, 1:1000), rabbit anti-PTEN (CST 9559, 1:1000), rabbit anti-INPP4B (Abcam ab81269, 1:1000), rabbit anti-pS6(S235/236) (CST 4858, 1:2000), mouse anti-S6 (CST 2317, 1:1000), rabbit anti-4EBP1(S65) (CST 9451, 1:1000), rabbit antipCRAF(S338) (CST 9427, 1:1000), rabbit anti-pMEK1/2(S217/221) (CST 9154, 1:1000), mouse anti-MEK1/2 (CST 4694, 1:1000), rabbit-anti-c-MYC (Abcam ab32072, 1:1000), rabbit anti-c-MYC (CST 5605, 1:1000), and rabbit anti-YAP1 (CST 4912, 1:1000). HSP90 and beta-Tubulin were used as loading controls. RASGTP assays were performed using an Active RAS Pull-Down and Detection Kit (Thermo Scientific 16117) per the manufacturer's instructions using in vitro GTP $\gamma \mathrm{S}$ (non-hydrolysable) and GDP pull-down controls and the provided panRAS antibody (1:200). Mouse RTK arrays (R\&D Systems) were assayed per the manufacturer's instructions. Primary antibodies were detected with fluorescent DyLight-conjugated (CST) or HRP-conjugated (BioRad) secondary antibodies for fluorescent (LI-COR) or chemiluminescent detection (Amersham), respectively. Approximate locations of protein size markers (in $\mathrm{kDa}$ ) are shown in individual blots. Quantification of protein levels from western blots was performed using Image Studio Lite (LI-COR).
Genomic DNA isolation and sequencing. Genomic DNA from cell lines was collected using QuickExtract DNA extraction solution (Epicentre) or a QiaAMP DNA Mini Kit (Qiagen). PCR products for sequencing were amplified using a Herculase II Fusion DNA polymerase kit (Agilent) and primers described in Supplementary Table 6. PCR products from cell lines were subject to Sanger sequencing (Quintara Biosciences). For subclones with multiple KRAS mutant alleles, PCR products were cloned into a TOPO vector (Life Technologies) and at least 10-20 bacterial colonies were sequenced per cell line. The most probable offtarget genes for sgRNAs were identified using CRISPR Design (http://crispr.mit. edu) and no gene had fewer than three exonic mismatches. All sequences and chromatograms were analyzed using MacVector software.

Genomic DNA was isolated from ground flash frozen tumor tissue using a High Pure PCR Template Preparation Kit (Roche). Mouse Kras exon 3 was PCR amplified using Herculase II Fusion DNA polymerase. Sequencing libraries were prepared from $50 \mathrm{ng}$ of PCR product using the Nextera DNA Sample Preparation Kit (Illumina) and sequenced with Illumina MiSeq. Sequencing reads (150 bp paired-end) were trimmed to $120 \mathrm{bp}$ after reviewing base quality profiles, in order to drop lower quality $3^{\prime}$ ends. Traces of Nextera adapters were clipped using the FASTX toolkit (Hannon Lab, CSHL) and pairs with each read greater than $15 \mathrm{bp}$ in length were retained. Additionally, read pairs where either read had $50 \%$ or more bases below a base quality threshold of Q30 (Sanger) were dropped from subsequent analyses. The reference sequence of the target locus was supplemented with $10 \mathrm{bp}$ genomic flanks and was indexed using an enhanced suffix array. Read ends were anchored in the reference sequence using $10 \mathrm{bp}$ terminal segments for a suffix array index lookup to search for exact matches. A sliding window of unit step size and a maximal soft-clip limit of $10 \mathrm{bp}$ was used to search for possible anchors at either end of each read. For each read, optimal Smith-Waterman dynamic programming alignment was performed between the reduced state space of the read sequence and the corresponding reference sequence spanning the maximally distanced anchor locations. Scoring parameters were selected to allow for sensitive detection of short and long insertions and deletions while allowing for up to four mismatches, and the highest scoring alignment was selected. Read pairs with both reads aligned in the proper orientation were processed to summarize the number of wild-type reads and the location and size of each insertion and deletion event. Overlapping reads within pairs were both required to support the event if they overlapped across the event location. Additionally, mutation events and wild-type reads were summarized within the extents of the sgRNA sequence and PAM site by considering read alignments that had a minimum of $20 \mathrm{bp}$ overlap with this region. Mutation calls were translated to genomic coordinates and subsequently annotated using Annovar (http://annovar.openbioinformatics.org). The alignment and post-processing code was implemented in $\mathrm{C}++$ along with library functions from SeqAn (https://www.seqan.de/) and SSW ${ }^{65}$ and utility functions in Perl and in the R statistical programming language (www.R-project.org). Mutation calls were subjected to manual review using the Integrated Genomics Viewer (IGV; http://software.broadinstitute.org/software/igv/).

Quantitative PCR for copy number analysis. Quantitative PCR of the KRAS locus and control regions (LINE1 for human, chromosome 5 10054507-10054621 region for mouse) was performed as previously described ${ }^{66,67}$ in triplicate using SYBR green reagents (Life Technologies) with primers listed in Supplementary Table 7. Genomic DNA from 293T and murine ES cells were used as control cells to determine $2 \mathrm{~N}$ copy number for human and mouse samples, respectively. $C_{\mathrm{p}}$ values were measured by a LightCycler 480 Real-Time PCR System (Roche). KRAS DNA relative to control region (KRAS $\left.{ }^{a m p}\right)$ was calculated for samples and control cell lines using the following formula: $2^{(K R A S C p-C o n t r o l ~ r e g i o n ~ C p) ~}$ KRAS copy number was determined using the following formulation: $2 \times K R A S^{a m p}$ (sample)/KRAS ${ }^{a m p}$ (control cell line).

High-throughput drug screening. High-throughput screening was performed as previously described ${ }^{68}$. To determine the optimal plating density during assay development, 8988T E3, E6, H9, and H36 cells were plated at either 500, 1000, or 1500 cells per well into 384 -well opaque, white assay plates (Corning), $50 \mu \mathrm{L}$ per well, and incubated overnight at $37^{\circ} \mathrm{C} / 5 \% \mathrm{CO}_{2}$. The next day, cells were treated with MG-132 (Enzo Bioscience) starting at a high concentration of $40 \mu \mathrm{M}$, in a 14$\mathrm{pt}$, twofold dilution series, 16 replicates/concentration, for $72 \mathrm{~h}$. $0.1 \%$ DMSO (solvent for all compounds) was used as a negative control. Sensitivity was assayed using CTG. Luminescence was measured using a M1000 Infinite Pro plate reader (Tecan). The $Z^{\prime}$ factor at each concentration point was calculated and compared between each cellular density to determine the largest dynamic detection window for subsequent screening. Estimated $\mathrm{Z}^{\prime}$ factors were calculated using the following formula: $1-\left(3 \times\left(\sigma_{\mathrm{p}}+\sigma_{\mathrm{n}}\right) /\left(\mu_{\mathrm{p}}-\mu_{\mathrm{n}}\right)\right)$ where $\sigma$ (standard deviation) and $\mu$ (mean) were determined from the positive (p) and negative (n) controls. For the screen, cells were plated at a density optimized during assay development as above. A modified version of the Selleck Cambridge Cancer Compound Library (http:// www.selleckchem.com/screening/cambridge-cancer-compound-library.html) containing 384 structurally diverse, medicinally active, and cell permeable cancerrelevant compounds was used for screening. Compounds were plated in 384-well format in 5-pt, 10-fold concentration ranges, starting at $10 \mathrm{mM}$. $50 \mathrm{~nL}$ of compounds were pin-transferred (V\&P Scientific pin tool mounted onto a Tecan Freedom Evo 150 MCA96 head) into duplicate assay plates and incubated for $72 \mathrm{~h}$ 
The DMSO content was $0.1 \%$ within each well. Thirty-two wells of DMSO vehicle control and 32 wells of positive control MG-132 were included on each plate. After 3 days of incubation, $10 \mu \mathrm{L}$ of CTG was added to each well, incubated for $10 \mathrm{~min}$, and luminescence output was read as a surrogate for cell viability. $\mathrm{Z}^{\prime}$ factors were $>0.5$ for all plates in the screen. Percent viability (PV) compared to DMSO control was calculated for each compound well and plotted against $\log _{10}$ (Dose) (M). Area under the curve (AUC) was calculated using the trapezoidal rule: $\left(\left(\mathrm{PV}_{1}+\mathrm{PV}_{2}\right) /\right.$ 2) $\times\left(\right.$ dose $_{1}-$ dose $\left._{2}\right)$. AUCs were averaged for intact $\left(\mathrm{AUC}_{\mathrm{i}}\right)$ and knockout $\left(\mathrm{AUC}_{\mathrm{KO}}\right.$ ) cells across replicates. Compounds were considered hits if (1) $\mathrm{AUC}_{\mathrm{i}}$ or $\mathrm{AUC}_{\mathrm{KO}}$ were $<4$; (2) $\triangle \mathrm{AUC}\left(\mathrm{AUC}_{\mathrm{i}}-\mathrm{AUC}_{\mathrm{KO}}\right)$ was $>0.5$ or $<-0.5$; and (3) $\mathrm{AUC}_{\mathrm{i}}$ and $\mathrm{AUC}_{\mathrm{KO}}$ were significantly different $(p<0.05$, Student's $t$ test)

Drug treatments. GDC-0941, BAY80-6946, AZD6244, MK2206, AZD8055, BYL719, TGX220, Idelalisib, BGJ-398, and Crenolanib were purchased from Selleck Chemical. Verteporfin was purchased from Sigma. All compounds were diluted to $10-50 \mathrm{mM}$ stock concentration in DMSO except for BAY80-6946, which was diluted to $5 \mathrm{mM}$ in DMSO with $10 \mathrm{mM}$ trifluoroacetic acid (TFA). To generate dose-response curves, cells (500-1000 for A13 clones, 2000-4000 for PANC-1 clones and primary hPDAC clones, and 1000-2000 for 8988T, KP-4, and MM1402 clones) were plated in 96-well white plates (Perkin Elmer) in $100 \mu \mathrm{L}$ of media and incubated overnight. Hundred microliter of drug at $2 \times$ final concentration was added to each well for 3-6 replicates for each cell line and dose. Cell viability was determined at $72 \mathrm{~h}$ using CTG. For ligand treatments, cells were treated with recombinant EGF, FGF1, or PDGF-BB (R\&D Systems) when initially plated. Percent viability was calculated for each dosed well compared to solvent controls (DMSO or DMSO with TFA at $0.1-0.2 \%$ ) and plotted against $\log _{10}$ (Dose) (M). For dose-response curves, each replicate for each cell line and dose was plotted along with curve fit regression for three-component inhibitor response (Prism). For longterm drug treatments, cells were plated at low-density in six-well plates in triplicate, treated with drugs for 10-14 days (media was refreshed with drug every 2 days), and stained with $0.5 \%$ crystal violet when control cells became confluent.

Animal studies. All animal studies were approved by the MIT Institutional Animal Care and Use Committee. A13 mouse clones were transplanted to form tumors in nude mice (Taconic) via subcutaneous injections. A total of $5 \times 10^{5}$ cells suspended in $100 \mu \mathrm{L}$ of cold PBS were injected per tumor to determine tumorforming capacity. For $8988 \mathrm{~T}$ and PANC-1 clones, $2 \times 10^{6}$ cells suspended in $100 \mu \mathrm{L}$ of cold 3:1 PBS:growth factor-reduced matrigel (Corning) were injected per tumor. For drug treatment of A13 tumors in vivo, a higher number of knockout cells $\left(2 \times 10^{6}\right)$ was injected to synchronize tumor formation between intact and knockout clones, as $5 \times 10^{5}$ cells of $\mathrm{K} 1$ and $\mathrm{K} 2$ give rise to subcutaneous tumors but at significantly slower rates than E1 and E2. Tumor formation was monitored over time by direct observation, caliper measurement, and IVIS spectrum optical imaging (Xenogen Corporation). When subcutaneous tumors grew to $0.5 \mathrm{~cm}$ in diameter (approximate bioluminescent radiance of $1 \times 10^{10}$ photons $\mathrm{s}^{-1} \mathrm{~cm}^{-2}$ per sr), mice were randomly dosed (based on ear tag number assigned at time of tumor transplant) with $150 \mathrm{mg} \mathrm{kg}^{-1}$ of GDC-0941 (LC Laboratories) or vehicle alone (10\% DMSO and 5\% Tween-20 in nuclease-free water) daily for 14 days by oral gavage. We estimated that to observe a 50\% reduction in tumor growth at 2 weeks after initiation of treatment (mean normalized volume \pm s.d. of $12 \pm 4$ ) with alpha of 0.05 and power of 0.8 , we would need at least seven tumors per group.

For transplant of A13 cells harboring DOX-inducible mmKras.366, $5 \times 10^{5}$ cells suspended in $100 \mu \mathrm{L}$ of cold PBS were injected per tumor subcutaneously. Clone 1 was injected to the left flank and clone 2 to the right flank of each immunocompromised mouse. DOX feed (Harlan-Teklad) was administered after all the tumors were $0.5 \mathrm{~cm}$ in diameter as monitored by caliper measurements. After 4 days of DOX feed, $150 \mathrm{mg} \mathrm{kg}^{-1}$ of GDC-0941 or vehicle alone was dosed daily for 14 days by oral gavage. Caliper measurements and bioluminescence imaging by IVIS was done in 3-4 day intervals by injecting $100 \mu \mathrm{l}$ of $30 \mathrm{mg} \mathrm{ml}^{-1}$ luciferin per mouse and imaging $10 \mathrm{~min}$ post injection. The level of bioluminescence in radiance was analyzed by Living Image software (Perkin Elmer). Tumor volume was calculated from caliper measurements using the modified ellipsoid formula: (length) $\times(\text { width })^{2} / 2$. Investigators were not blinded to treatment groups during protocol. Bliss independence for additive effect of DOX-induced KRAS knockout and GDC-0941 treatment was calculated for each measured time point using the following formulas: Fractional response to GDC-0941 $\left(\mathrm{F}_{\mathrm{GDC}}\right)=\left(1-\left(\mathrm{TV}_{\mathrm{GDC}} / \mathrm{TV}_{\mathrm{Veh}}\right)\right)$, Fractional response to DOX-induced KRAS knockout $\left(\mathrm{F}_{\mathrm{DOX}}\right)=\left(1-\left(\mathrm{TV}_{\mathrm{DOX}} / \mathrm{TV}_{\mathrm{Veh}}\right)\right)$, additive fractional response to GDC-0941 and DOX $\left(\mathrm{F}_{\mathrm{GDC}+\mathrm{DOX}}\right)=\mathrm{F}_{\mathrm{GDC}}+\mathrm{F}_{\mathrm{DOX}}-\left(\mathrm{F}_{\mathrm{GDC}} \times \mathrm{F}_{\mathrm{DOX}}\right)$. Bliss line corresponds to tumor volume (TV) with predictive additive effect of treatments and determined with the following equation at each time point: $\mathrm{TV}_{\mathrm{Veh}}\left(1-\mathrm{F}_{\mathrm{GDC}}+\mathrm{DOX}\right)$.

RNA isolation and sequencing analysis. RNA was isolated from PDAC cells using TRIzol (Life Technologies). cDNA libraries were prepared using an Illumina TruSeq sample preparation kit with indexed adapter sequences and polyA selection. Sequencing was performed on an Illumina HiSeq 2000 instrument to obtain single-end 50-nt reads. All reads that passed quality metrics were mapped to the UCSC mm9 mouse or hg19 human genome build (http://genome.ucsc.edu/) using RSEM (v1.2.12) (http://deweylab.github.io/RSEM/). For pairwise differential expression analyses, data normalization (MedianNorm) and differential analyses between experimental conditions were performed using EBSeq v1.4.0 (http:// bioconductor.org/packages/release/bioc/html/EBSeq.html). All RNA-Seq analyses were conducted in R. Unsupervised clustering was performed using a Pearson correlation-based pairwise distance measure. Heat maps were generated using the Heatplus package in $\mathrm{R}$.

High-resolution signature analyses between clones within each cell line were performed using a blind source separation methodology based on ICA ${ }^{69}$. RSEM generated estimated expression counts were upper quartile normalized to a count of 1000. The R implementation of the core JADE algorithm (Joint Approximate Diagonalization of Eigenmatrices) ${ }^{70}$ was used along with custom R utilities. Signatures were visualized using the sample-to-signature correspondence schematic afforded by Hinton plots, where colors represent relative directionality of gene expression (red relatively upregulated, blue relatively downregulated within each signature) and the size of each rectangle quantifies the strength of a signature (column) in a given sample (row). Each signature is two-sided, allowing for identification of upregulated and downregulated genes for each signature within each sample. Biologically relevant and statistically significant signatures were identified using a Mann-Whitney $U$-test. Signature correlation scores ( $z$-scores) for each gene in the statistically significant signatures are included as supplementary tables. Heat maps were plotted with the top and bottom $2 \%$ genes in each signature. Additionally, genes with standardized signature correlation scores $z>3$ (alternatively $z<-3$ ) were used as gene sets to score TCGA (https://tcga-data.nci. nih.gov/tcga/) Pancreatic adenocarcinoma (PAAD) tumors using ssGSEA. Tumors were stratified based on standardized signature scores $(z$-scores) derived using ssGSEA and associated patients within matching top and bottom percentile buckets were compared for a statistically significant difference in survival times. Significance of overlap between tumor buckets scored using 8988T, A13, combined knockout, quasi-mesenchymal ${ }^{21}$, basal ${ }^{46}$, and squamous ${ }^{45}$ subtype signatures was assessed using the hypergeometric test. Survival analyses were conducted using the survival package in $\mathrm{R}$.

In order to derive a combined knockout signature, the top and bottom $2 \%$ genes in the human signature were analyzed in the mouse expression data set and ICA analysis revealed a WT/KO signature. The up and down gene sets were determined using a standardized signature correlation score of $z>0.5$ (alternatively, $z<-0.5$ ) These were used in survival analyses similar to those described above for TCGA and also for ICGC Pancreatic Cancer Australia (PACA-AU) tumors (https://icgc. $\mathrm{org} / \mathrm{icgc} / \mathrm{cgp} / 68 / 304 / 798)$.

Publicly available microarray data sets were used to generate KRAS-ON and KRAS-OFF signatures from a mouse model of DOX-regulated KRAS transgene expression $^{22,39,40}$. Array CEL files were retrieved from GEO (GSE32277,

GSE53169, and GSE58307) and processed using Affymetrix Power Tools v. 1.15.0 (rma-sketch). Probes were collapsed (max. value) to yield per gene expression estimates. Genes with upper quartile $\log _{2}$ expression value less than 5 across all samples were dropped from further analysis. The resulting data sets were used for signature analysis with ICA.

Publicly available RNA-Seq data sets were used to generate the CTC signature ${ }^{44}$. Read counts for the sample set (number of raw reads mapped per gene) were downloaded from GEO for record GSE51372. Entries with duplicate symbols or missing gene names were dropped from further consideration. Samples with less than 5 million total mapped reads were dropped from the data set in order to eliminate expression noise from low coverage. Only samples identified as tumor or classical CTC were retained for downstream analyses. Read counts for the remaining samples were normalized using quartile normalization with the upper quartile set to 1000 . In the resulting expression data set, genes with an upper quartile of expression count less than 1000 across all samples were tagged as lowly expressed genes and dropped. Normalized expression values were $\log _{2}$ transformed and used as input for signature analysis using ICA.

GSEA were carried out using the pre-ranked mode using $\log _{2}$ fold-change values (for pairwise analyses) or standardized signature correlation scores (for ICA signatures) with default settings ${ }^{38}$. Network representations of GSEA results were generated using EnrichmentMap (http://www.baderlab.org/Software/ EnrichmentMap) for Cytoscape v3.3.0 (http://www.cytoscape.org) with $p$ value and FDR cutoffs as described in figure legends. Each circle represents a gene set with circle size corresponding to gene set size and intensity corresponding to enrichment significance. Red is upregulated and blue is downregulated. Each line corresponds to minimum $50 \%$ mutual overlap with line thickness corresponding to degree of overlap. Cellular processes for gene set clusters were manually curated.

Candidate point mutations in RNA-Seq data sets were called using a pipeline based on the GATK Toolkit (https://software.broadinstitute.org/gatk/).

Transcriptomic reads were mapped (to $\mathrm{mm} 9$, hg19) using the Tophat (v2.0.4) spliced aligner and subjected to local realignment and score recalibration using the GATK Toolkit. Mutations were called in KO samples (individual and pooled) against WT samples (individual and pooled) with a minimum base quality threshold of 30. Genomic annotations were performed using ANNOVAR (http:// www.openbioinformatics.org/annovar/).

Statistical analyses. $P$ values for comparisons of two groups were determined by two-tailed Student's $t$ test (for normally distributed data) or Mann-Whitney $U$-test (for non-parametric data) as noted in the figure legends. All replicates were included in these analyses. The log-rank test was used for Kaplan-Meier survival 
analyses. The Cox regression model (in univariate and multivariable settings) was used to estimate hazard ratios in survival analyses. A $p$ value of $<0.05$ was used to denote statistical significance. All error bars denote standard error of mean (s.e.m.) or standard deviation (s.d.) as noted in the figure legends.

Code availability. Computer code for RNA-Seq independent component analyses is available upon request. Other software tools for RNA-Seq analyses, website source, and version numbers are listed above.

Data availability. The RNA and DNA sequencing data sets that support the findings of this study have been deposited in the Gene Expression Omnibus (GEO) and the NCBI Sequencing Read Archive (SRA) under accession GSE71876. Previously published data sets used in this study are available in GEO under accession GSE32277, GSE53169, GSE58307, and GSE51372. The authors declare that all other data are available within the article and its supplementary information files or available from the corresponding author upon request.

Received: 18 June 2017 Accepted: 7 August 2017

Published online: 23 October 2017

\section{References}

1. American Cancer Society. Cancer Facts \& Figures 2017 (American Cancer Society, Atlanta, 2017).

2. Ryan, D. P., Hong, T. S. \& Bardeesy, N. Pancreatic adenocarcinoma. NEJM 371 , 1039-1049 (2014).

3. Conroy, T. et al. FOLFIRINOX versus gemcitabine for metastatic pancreatic cancer. N. Engl. J. Med. 364, 1817-1825 (2011).

4. Von Hoff, D. D. et al. Increased survival in pancreatic cancer with nabpaclitaxel plus gemcitabine. N. Engl. J. Med. 369, 1691-1703 (2013).

5. Hezel, A. F., Kimmelman, A. C., Stanger, B. Z., Bardeesy, N. \& DePinho, R. A. Genetics and biology of pancreatic ductal adenocarcinoma. Genes Dev. 20, 1218-1249 (2006).

6. Jones, S. et al. Core signaling pathways in human pancreatic cancers revealed by global genomic analyses. Science 321, 1801-1806 (2008).

7. Biankin, A. V. et al. Pancreatic cancer genomes reveal aberrations in axon guidance pathway genes. Nature 491, 399-405 (2012).

8. Waddell, N. et al. Whole genomes redefine the mutational landscape of pancreatic cancer. Nature 518, 495-501 (2015).

9. Karnoub, A. E. \& Weinberg, R. A. Ras oncogenes: split personalities. Nat. Rev. Mol. Cell. Biol. 9, 517-531 (2008).

10. Stephen, A. G., Esposito, D., Bagni, R. G. \& McCormick, F. Dragging ras back in the ring. Cancer Cell 25, 272-281 (2014).

11. Hingorani, S. R. et al. Preinvasive and invasive ductal pancreatic cancer and its early detection in the mouse. Cancer Cell 4, 437-450 (2003).

12. Ostrem, J. M., Peters, U., Sos, M. L., Wells, J. A. \& Shokat, K. M. K-Ras(G12C) inhibitors allosterically control GTP affinity and effector interactions. Nature 503, 548-51 (2013).

13. Lito, P., Solomon, M., Li, L., Hansen, R. \& Rosen, N. Allele-specific inhibitors inactivate mutant KRAS G12C by a trapping mechanism. Science 351, 604-608 (2016).

14. Maurer, T. et al. Small-molecule ligands bind to a distinct pocket in Ras and inhibit SOS-mediated nucleotide exchange activity. Proc. Natl Acad. Sci. 109, 5299-5304 (2012)

15. Sun, Q. et al. Discovery of small molecules that bind to K-Ras and inhibit Sos-mediated activation. Angew. Chem. Int. Ed. 51, 6140-6143 (2012).

16. Zimmermann, G. et al. Small molecule inhibition of the KRAS-PDES interaction impairs oncogenic KRAS signalling. Nature 497, 638-42 (2013).

17. Ledford, H. The ras renaissance. Nature 520, 278-280 (2015).

18. Cox, A. D., Fesik, S. W., Kimmelman, A. C., Luo, J. \& Der, C. J. Drugging the undruggable RAS: mission possible? Nat. Rev. Drug Discov. 13, 828-51 (2014).

19. Brummelkamp, T. R., Bernards, R. \& Agami, R. Stable suppression of tumorigenicity by virus-mediated RNA interference. Cancer Cell 2, 243-247 (2002).

20. Singh, A. et al. A gene expression signature associated with 'K-Ras addiction' reveals regulators of EMT and tumor cell survival. Cancer Cell 15, 489-500 (2009).

21. Collisson, E. A. et al. Subtypes of pancreatic ductal adenocarcinoma and their differing responses to therapy. Nat. Med. 17, 500-503 (2011).

22. Kapoor, A. et al. Yap1 activation enables bypass of oncogenic KRAS addiction in pancreatic cancer. Cell 158, 185-197 (2014).

23. Ran, F., Hsu, P., Wright, J. \& Agarwala, V. Genome engineering using the CRISPR-Cas9 system. Nat. Protoc. 8, 2281-308 (2013).

24. Shi, J. et al. Discovery of cancer drug targets by CRISPR-Cas9 screening of protein domains. Nat. Biotechnol. 33, 661-667 (2015).

25. Aguirre, A. J. et al. Genomic copy number dictates a gene-independent cell response to CRISPR-Cas9 targeting. Cancer Discov. 6, 914-29 (2016).
26. Shao, D. D. et al. KRAS and YAP1 converge to regulate EMT and tumor survival. Cell 158, 171-184 (2014).

27. Liu-chittenden, Y. et al. Genetic and pharmacological disruption of the TEAD YAP complex suppresses the oncogenic activity of YAP. Genes Dev. 26, 1300-1305 (2012).

28. Turke, A. B. et al. MEK inhibition leads to PI3K/AKT activation by relieving a negative feedback on ERBB receptors. Cancer Res. 72, 3228-3237 (2012).

29. Ebi, H. et al. Receptor tyrosine kinases exert dominant control over PI3K signaling in human KRAS mutant colorectal cancers. J. Clin. Invest. 121, 4311-4321 (2011).

30. Lee, H. J. et al. Drug resistance via feedback activation of stat3 in oncogeneaddicted cancer cells. Cancer Cell 26, 207-221 (2014).

31. Ebi, $\mathrm{H}$. et al. PI3K regulates MEK/ERK signaling in breast cancer via the Rac-GEF, P-Rex1. Proc. Natl Acad. Sci. USA 110, 21124-9 (2013).

32. Will, M. et al. Rapid induction of apoptosis by PI3K inhibitors is dependent upon their transient inhibition of RAS-ERK signaling. Cancer Discov. 4, 334-348 (2014)

33. She, Q. et al. 4E-BP1 is a key effector of the oncogenic activation of the AKT and ERK signaling pathways that integrates their function in tumors. Cancer Cell 18, 39-51 (2012)

34. Martineau, Y. et al. Pancreatic tumours escape from translational control through 4E-BP1 loss. Oncogene 33, 1367-74 (2014).

35. Ilic, N., Utermark, T., Widlund, H. R. \& Roberts, T. M. PI3K-targeted therapy can be evaded by gene amplification along the MYC-eukaryotic translation initiation factor 4E (eIF4E) axis. Proc. Natl Acad. Sci. USA 108, E699-E708 (2011).

36. Wendel, H.-G. et al. Survival signalling by Akt and eIF4E in oncogenesis and cancer therapy. Nature 428, 332-337 (2004).

37. Mootha, V. K. et al. PGC-1alpha-responsive genes involved in oxidative phosphorylation are coordinately downregulated in human diabetes. Nat. Genet. 34, 267-273 (2003).

38. Subramanian, A., Tamayo, P., Mootha, V. K., Mukherjee, S. \& Ebert, B. L. Gene set enrichment analysis: A knowledge-based approach for interpreting genomewide. Proc. Natl Acad. Sci. USA 102, 15545-15550 (2005).

39. Ying, $\mathrm{H}$. et al. Oncogenic kras maintains pancreatic tumors through regulation of anabolic glucose metabolism. Cell 149, 656-670 (2012).

40. Viale, A. et al. Oncogene ablation-resistant pancreatic cancer cells depend on mitochondrial function. Nature 514, 628-632 (2014).

41. Seguin, L. et al. An integrin $\beta 3$-KRAS-RalB complex drives tumour stemness and resistance to EGFR inhibition. Nat. Cell Biol. 16, 457-68 (2014).

42. DeNicola, G. M. et al. Oncogene-induced Nrf2 transcription promotes ROS detoxification and tumorigenesis. Nature 475, 106-109 (2011).

43. Whittle, M. C. et al. RUNX3 controls a metastatic switch in pancreatic ductal adenocarcinoma. Cell 161, 1345-1360 (2015).

44. Ting, D. T. et al. Single-cell RNA sequencing identifies extracellular matrix gene expression by pancreatic circulating tumor cells. Cell Rep. 8, 1905-1918 (2014)

45. Bailey, P. et al. Genomic analyses identify molecular subtypes of pancreatic cancer. Nature 531, 47-52 (2016)

46. Moffitt, R. A. et al. Virtual microdissection identifies distinct tumor- and stroma-specific subtypes of pancreatic ductal adenocarcinoma. Nat. Publ. Gr. 47, 1168-1178 (2015).

47. Eser, S. et al. Selective requirement of PI3K/PDK1 signaling for kras oncogenedriven pancreatic cell plasticity and cancer. Cancer Cell 23, 406-420 (2013).

48. Payne, S. N. et al. PIK3CA mutations can initiate pancreatic tumorigenesis and are targetable with PI3K inhibitors. Oncogenesis 4, e169 (2015).

49. Hofmann, I. et al. K-RAS mutant pancreatic tumors show higher sensitivity to MEK than to PI3K inhibition in vivo. PLoS ONE 7, e44146 (2012).

50. Junttila, M. R. et al. Modeling targeted inhibition of MEK and PI3 kinase in human pancreatic cancer. Mol. Cancer Ther. 14, 40-47 (2014).

51. Alagesan, B. et al. Combined MEK and PI3K inhibition in a mouse model of pancreatic cancer. Clin. Cancer Res. 21, 396-404 (2015).

52. Wells, V., Downward, J. \& Mallucci, L. Functional inhibition of PI3K by the betaGBP molecule suppresses Ras-MAPK signalling to block cell proliferation. Oncogene 26, 7709-7714 (2007).

53. Carracedo, A. et al. Inhibition of mTORC1 leads to MAPK pathway activation through a PI3K-dependent feedback loop in human cancer. J. Clin. Invest. 118, 3065-3074 (2008).

54. Diaz-Flores, E. et al. PLC- and PI3K link cytokines to ERK activation in hematopoietic cells with normal and oncogenic Kras. Sci. Signal. 6, ra105-ra105 (2013).

55. Akutagawa, J. et al. Targeting the PI3K/Akt pathway in murine MDS/MPN driven by hyperactive Ras. Leukemia 30, 1335-1343 (2016)

56. Sampaio, C. et al. Signal strength dictates phosphoinositide 3-kinase contribution to Ras/extracellular signal-regulated kinase 1 and 2 activation via differential Gab1/Shp2 recruitment: consequences for resistance to epidermal growth factor receptor inhibition. Mol. Cell. Biol. 28, 587-600 (2008).

57. Makohon-Moore, A. P. et al. Limited heterogeneity of known driver gene mutations among the metastases of individual pancreatic cancer patients (accepted). Nat. Genet. 49, 358-366 (2017). 
58. McDonald, O. G. et al. Epigenomic reprogramming during pancreatic cancer progression links anabolic glucose metabolism to distant metastasis. Nat. Genet. 49, 367-376 (2017).

59. Noll, E. M. et al. CYP3A5 mediates basal and acquired therapy resistance in different subtypes of pancreatic ductal adenocarcinoma. Nat. Med. 22, 278-287 (2016).

60. Friedlander, S. Y. G. et al. Context-dependent transformation of adult pancreatic cells by oncogenic K-ras. Cancer Cell 16, 379-389 (2009).

61. Hingorani, S. R. et al. Trp53R172H and KrasG12D cooperate to promote chromosomal instability and widely metastatic pancreatic ductal adenocarcinoma in mice. Cancer Cell 7, 469-83 (2005).

62. Sanjana, N. E., Shalem, O. \& Zhang, F. Improved vectors and genome-wide libraries for CRISPR screening. Nat. Protoc. 11, 783-784 (2014).

63. Aubrey, B. J. et al. An inducible lentiviral guide RNA platform enables the identification of tumor-essential genes and tumor-promoting mutations in vivo. Cell Rep. 10, 1422-1432 (2015).

64. Akama-garren, E. H. et al. A modular assembly platform for rapid generation of DNA constructs. Sci. Rep. 6, 16836 (2015).

65. Zhao, M., Lee, W.-P., Garrison, E. \& Marth, G. An SIMD Smith-Waterman $\mathrm{C} / \mathrm{C}++$ library for use in genomic applications. PLoS ONE 8, e82138 (2013).

66. Modrek, B. et al. Oncogenic activating mutations are associated with local copy gain. Mol. Cancer Res. 7, 1244-1253 (2009).

67. Sánchez-Rivera, F. J. et al. Rapid modelling of cooperating genetic events in cancer through somatic genome editing. Nature 516, 428-431 (2014).

68. Basu, A. et al. An interactive resource to identify cancer genetic and lineage dependencies targeted by small molecules. Cell 154, 1151-1161 (2013).

69. Hyvarinen, A. \& Oja, E. Independent component analysis: algorithms and applications. Neural Netw. 13, 411-430 (2000).

70. Rutledge, D. N. \& Jouan-Rimbaud Bouveresse, D. Independent components analysis with the JADE algorithm. Trends Anal. Chem. 50, 22-32 (2013).

\section{Acknowledgements}

We thank Jeffrey Settleman for critical reading of the manuscript; V. Gocheva, N. Dimitrova, F. Sanchez-Rivera, C. Li, K. Chung, C. Ellis, B. Wagner, A. Aguirre, and O. Shalem for technical assistance and reagents; J. Cheah and C. Soule from the Koch Institute (KI) Genomics Core/HTS Facility for screening assistance; K. Cormier and C. Condon from the Hope Babette Tang (1983) Histology Facility for histology assistance; M. Jennings, M. Griffin, G. Paradis and S. Malstrom from the Swanson Biotechnology Center for technical support; S. Levine and A. Perez from the MIT BioMicro Center for RNA-Seq support; and W. Hahn, M. Herold, S. Lowe, W. Sellers, R. Weinberg and F. Zhang for constructs. We acknowledge that all experimental and analytical work done in the KI Genomics Core/HTS Facility was funded by the NIH and must be made available through the NIH's public access policy when published (https://publicaccess. nih.gov/). This work was supported by the Howard Hughes Medical Institute, Lustgarten Foundation Consortium Grant and Research Investigator Award, Blum-Kovler Pancreatic Cancer Action Network-AACR Innovative grant, Department of Defense Congressionally-Directed Medical Research Program grant (W81XWH-12-043), and in part by the Cancer Center Support (core) Grant P30-CA14051 from the National Cancer Institute (NCI). M.D.M. is supported by an NCI Mentored Clinical Scientist Research Career Development Award (K08-CA2080016-01) and was supported by a KL2/Catalyst Medical Research Investigator Training award (an appointed KL2 award) from Harvard Catalyst I The Harvard Clinical and Translational Science Center (National Center for Research Resources and the National Center for Advancing Translational Sciences), National Institutes of Health Award KL2 TR001100), and a Conquer Cancer Foundation-American Society for Clinical Oncology (CCF-ASCO) Young Investigator Award. The content is solely the responsibility of the authors and does not necessarily represent the official views of Harvard Catalyst, Harvard University and its affiliated academic healthcare centers, or the National Institutes of Health. T.J. is a Howard Hughes Medical Institute Investigator, the David H. Koch Professor of Biology, and a Daniel K. Ludwig Scholar.

\section{Author contributions}

M.D.M., P.-Y.C. and T.J. designed the study; M.D.M., P.-Y.C., K.J.D., K.M.C. and E.H performed experiments; A.B. conducted bioinformatics analyses; E.M.N., M.R.S. and A.T provided primary patient-derived PDAC cell lines; M.D.M., P.-Y.C. and T.J. wrote the manuscript with comments from all authors.

\section{Additional information}

Supplementary Information accompanies this paper at doi:10.1038/s41467-017-00942-5.

Competing interests: The authors declare no competing financial interests.

Reprints and permission information is available online at http://npg.nature.com/ reprintsandpermissions/

Publisher's note: Springer Nature remains neutral with regard to jurisdictional claims in published maps and institutional affiliations.

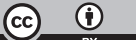

Open Access This article is licensed under a Creative Commons Attribution 4.0 International License, which permits use, sharing, adaptation, distribution and reproduction in any medium or format, as long as you give appropriate credit to the original author(s) and the source, provide a link to the Creative Commons license, and indicate if changes were made. The images or other third party material in this article are included in the article's Creative Commons license, unless indicated otherwise in a credit line to the material. If material is not included in the article's Creative Commons license and your intended use is not permitted by statutory regulation or exceeds the permitted use, you will need to obtain permission directly from the copyright holder. To view a copy of this license, visit http://creativecommons.org/ licenses/by/4.0/

(C) The Author(s) 2017 